Distribution Categories

Instruments (UC-37)

Engineering and Equipment (UC-38)

ANL-78-14

ARGONNE INATIONAL LABORATORY

9700 South Cass Avenue

Argonne, Illinois 60439

\title{
METHODS AND MEANS FOR \\ CONTROL OF ERROR IN \\ PRECISION INSTRUMENTS AND SYSTEMS
}

by

Robert N. Lewis

Electronics Division

February 1978

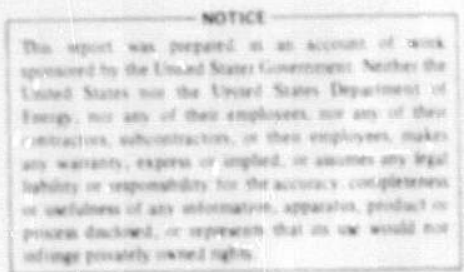




\section{PROLOGUE}

"But I wanted the magnetic field strength to go from 800.12 gauss to 812.74 gauss fast," said the complaining rabbit. "Well," said Alice, "I have done it. Look at the gaussmeter! It is fast, it only took 29 seconds to get there." "Well," said the despondent rabbit, "clearly vou have missed the point azain. It was at the correctvalue in 9 seconds but it overshot, reaching a peak in 12 seconds, and then played around until it finally was stable enough to get a reading at 29 seconds." The rabbit was clearly agitated.

"Why," he demanded, "don't you make it stop sooner and stay there? I only want it to stay there for 5 seconds before I want it to go somewhere else. Most of the time is wasted." "But," said Alice, somewhat morosely, "anything I do makes it worse. When I speed it up it almost becomes unstable; when I slow it down it takes even longer. This is the best I can do."

"I think," said the rabbit, rather severely, "that if you thought long enough and hard enough, you could do much better." 


\section{TAELE OF CONTENTS}

Page

NOMENCLATURE

ABSTRACT

1. INTRODUCTION . . . . . . . . . . . . . . . . .

11. THE RESPONSE OF PHYSICAL SYSTEMS ............. 11

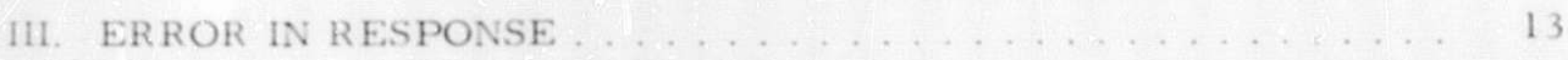

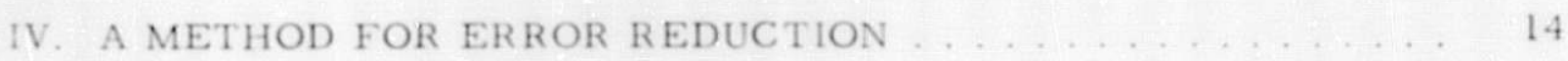

V. APPLICATION TO AN ELEMENTARY POSITIONAL SYSTEM ... 15

A. Open-loop Error Correction. . . . . . . . . . . 15

1. Application of the Method for Error Reduction . . . . . . 16

2. Method for Improving Response Time . . . . . . . . . . 20

3. Significant Overall improvement in System Performance. 22

B. Closed-loop Error Correction ... . . . . . . . . . 23

1. Some Additional Factors . . . . . . . . . . . . . . . 24

2. Positional Control .............................. 24

3. Positional and Velocity Control ...................... 25

C. Methods for Obtaining System Constants. . . . . . . . . . 27

1. Step-response Method. . . . . . . . . . . . . . . . 27

2. Sinusoidal-response Methods ................ 28

3. Summary of Describing Functions for $F_{0}(s)$ System ..... 28

VI. GENERAL APPLICATION. ............................. 31

A. System Elements and Modeling .................... 31

B. First-order Systems .............................. 32

C. More Complex Systems . . . . . . . . . . . . . . . . 32

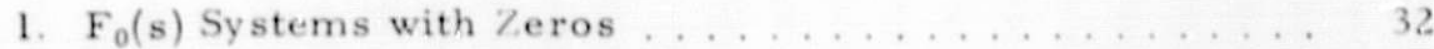

2. $F_{0}(s)$ System with Real Poles........................ 35

3. Correction of Complex Systems .............. 36

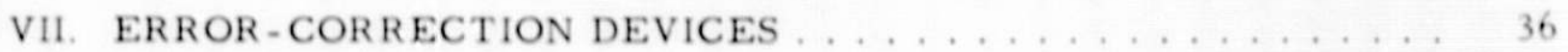

A. Real-pole and Real-zero Correction ............... 36 
TABLE OF CONTENTS

$\underline{\text { Page }}$

B. Correction Unit for $F_{0}(s)$ and $1 / F_{0}(s)$ Systems ........... 36

C. Portable Units for Determination of Error-correction Requirements for Systems ....................... 38

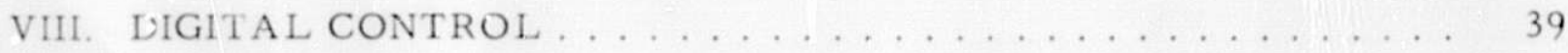

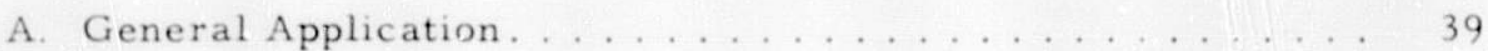

B. Sequential Control Programs..................... 39

IX. ADP.PTIVE SONTROL THROUGH USE OF PREDICTION

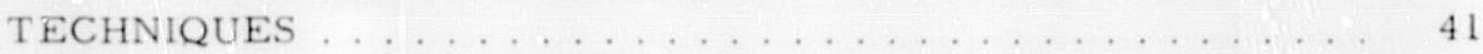

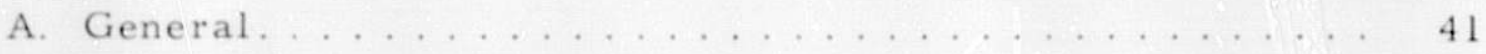

E. Marginally Stable Systeris ................. 43

C. Large-signal Error Control .................. 44

X. NOISE-GENERATED ERRORS IN SYSTEM RESPONSE . . . . 45

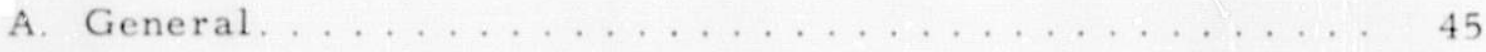

B. Mean-square Error. ....................... 45

C. Method for the Reduction of System Output Error due to Internal Noise. . . . . . . . . . . . . . . . 47

1. Application ........................ 47

2. Method .......................... 48

3. Results .............................. 50

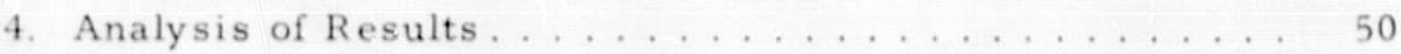

5. Discussion of Results ................ 53

APPENDIX: Effect of Pole and Zero Locations on Time Response of

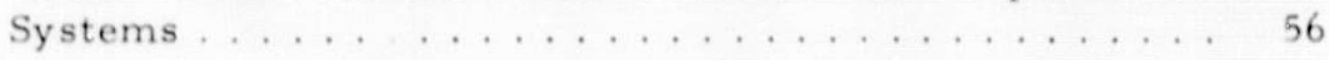

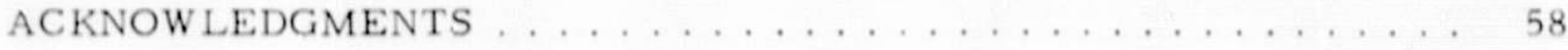

REFERENCES . . . . . . . . . . . . . . . . . . . . . . . . . . . . . . 58 


\section{LIST OF FIGURES}

No.

1. A Method for Output-error Reduction ............. 15

2. Connection of Error-correction Unit to an Open-ioop System. . . 16

3. Electrical Circuit Having Characteristics of Elementary

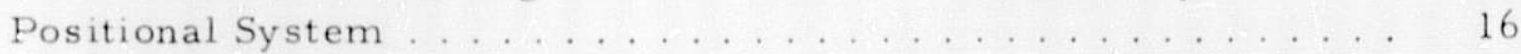

4. Time Response of $F_{0}(s)$ System to Delta, Step, Velocity, and Acceleration Inputs

5. Method for Providing Correctional Input, o(s). for the $F_{0}(s)$ System.

6. Response of $F_{0}(s)$ System to an Input Signal $E(t)+\rho(t)=$ $\frac{1}{2} a t^{2}+a+t+a / \omega_{n}^{2}$

7. Response of $F_{0}(s)$ System to an Input Signal $E(t)+\rho(t)=$ $\mathrm{Vt}+\mathrm{V}_{\tau}+\mathrm{V} /\left.\mathrm{\omega}_{\mathrm{n}}^{2}\right|_{\mathrm{s}}+$

8. General Requirement for Smooth Step-function Response in Time Interval Ts .

9. Input Program for Step-positional Response. . . . . . . . . . 20

10. Concrol Device for Improved Open-loop Control of $\mathrm{F}_{0}(\mathrm{~s})$ System. . 20

11. Improvement of System Performance ................ 22

12. Connection of Error-correction System to a Closed-loop System. 23

13. Definition of Additional Factors $\gamma$ and $\beta \ldots \ldots \ldots 24$

14. Positional Error Control for Elementary System. . . . . . . . . 25

15. Programming of Positional Error Correction for Elementary

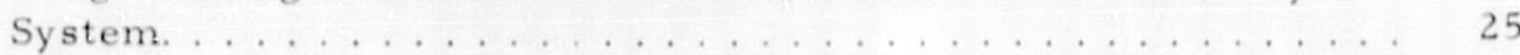

16. Error Correction for Position and Vslocity for Elementary

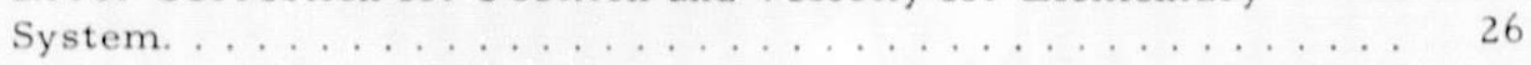

17. Possible Error Correction for High-voltage Power Supply . . . . 26

18. Possible Mechanization for Positional and Velocity Error

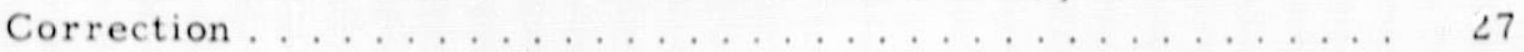

19. Derivation of Formulas for Calculating Values Shown in Table II

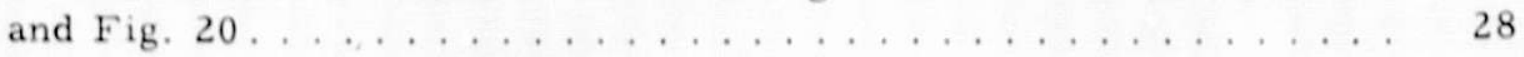

20. Parameters for $F_{0}(s)$ System when $\Psi_{1}$ and $T_{S}$ Are Known ..... 29

21. Determination of System Constants from Sinusoidal Response

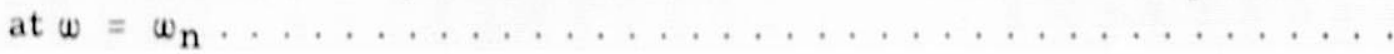




\section{LIST OF FIGURES}

No.

Title

$\underline{\text { Page }}$

22. Determination of System Constants from Peak Sinusoidal

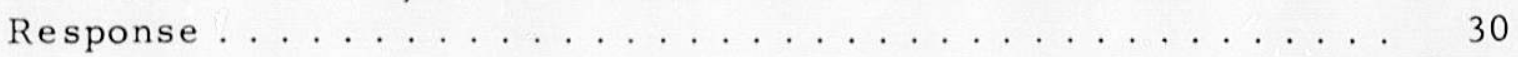

23. Some Basic System Elements.................. 31

24. System Modeling with Basic Elements............. 31

25. Error Correction for the $\mathrm{p} /(\mathrm{s}+\mathrm{p})$ First-order System. . . . . 32

26. Step Response of the $F_{0}(s)$ System with Zeros........... 34

27. Step Response of the Velocity-corrected Positional System When Using Control Techniques...................... 34

28. Overall Correction for $F(s)=\pi[(s+z) / z] F_{0}(s)$ System. . . . . 35

29. Method for Converting $\pi[\mathrm{p} /(\mathrm{s}+\mathrm{p})] \mathrm{F}_{0}(\mathrm{~s})$ System Response to That of the $\mathrm{F}_{0}(\mathrm{~s})$ Response...................... 36

30. Pole- and Zero-correcting Unit .................. 37

31. Unit for Correcting Either $\mathrm{F}_{0}(\mathrm{~s})$ or $1 / \mathrm{F}_{0}(\mathrm{~s})$ System ....... 37

32. Unit for Correcting Either $F_{0}(s)$ or $1 / F_{0}(s)$ System without Step

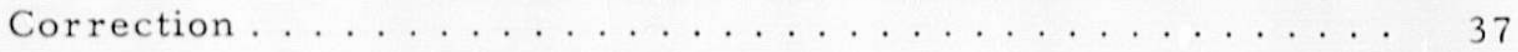

33. Unit for Correcting Either $F_{0}(s)$ or $1 / F_{0}(s)$ System with Step

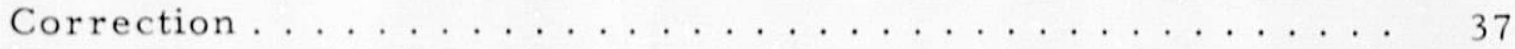

34. Portable Test Unit for Determining Optimum Correction Programs for Systems . . . . . . . . . . . . . . . .

35. Possible System for Error Correction of $F_{0}(s),[(s+z) / z] F_{0}(s)$,

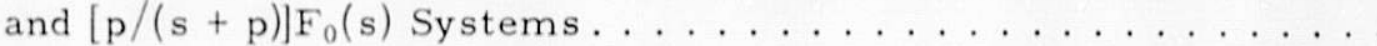

36. Sequential Control Program for Step Control of a Complex System. . . . . . . . . . . . . . . . . . 40

37. Analysis of Adaptive Control of Temperature of Liquid by Timeshared Digital Computer.....................

38. Time History of Adaptive Temperature Control. . . . . . . . . . . 43

39. Possible Error Monitor and Control Unit to Operate with DAS. . 44

40. Large-signal Error Control. . . . . . . . . . . . . . . . . . . . 44

41. Typical Examples of Noise Spectral Densities. . . . . . . . . . . 45

42. Inclusion of Error-correction Unit (ECU) in Feedback Path. . . . . 48

43. Block Diagram of Error-correction Unit . . . . . . . . . . . . . 49

44. Schematic Diagram of Error-correction Unit . . . . . . . . . . 51 


\section{LIST OF FIGURES}

No.

Title

Page

45. Recording of Amplified Error Signal of Precision Calorimeter . . 52

46. Impulse Response of the $\mathrm{F}_{0}(\mathrm{~s})$ System . . . . . . . . . . . 53

A.1. s-plane Geometry and Step Response for Several Systems. . . . . 56

\section{LIST OF TABLES}

No.

$\underline{\text { Title }}$

$\underline{\text { Page }}$

I. Unit-step Response for $\mathrm{F}_{0}(\mathrm{~s})=\mathrm{A} /\left(\mathrm{s}^{2}+10 \mathrm{~s}+\mathrm{A}\right) \ldots \ldots 21$

II. Describing Parameters for the $F_{0}(s)$ System. . . . . . . . . . 29

III. Characteristics of the Velocity-corrected Positional System . . . 34

IV. Noise Response of the $\mathrm{F}_{0}(\mathrm{~s})$ System. . . . . . . . . . . . . 47 
NOMENCLAT URE

Symbol

a Acceleration input signal, e.g., $\frac{1}{2} a t^{2}$

|A| Absolute-value amplifier (Fig. 42)

$\alpha \quad$ Logarithmic damping coefficient, e.g., $\mathrm{e}^{-\alpha \mathrm{t}}$ (Fig. 3)

A Definition of $e^{-\Delta t / R C} /$ Fig. 36)

8 Ratio, $\frac{\text { Magnitude of system output at } \mathrm{T}_{\mathrm{S}}}{\text { Value of input, } \frac{1}{2} \mathrm{aT}_{\mathrm{S}}^{2}}$

C Electrical capacitance

6. System constant $\delta=\alpha / \omega$ (Fig. 3)

$6^{+} \quad$ Impulse input signal

E Electrical voltage

$E_{0}(s) \quad$ Laplace-transformed output of a system

$E_{\text {in }}(s)$ Laplace-transformed input of a system

ECU Error-correction unit

e Error signal

$\dot{\varepsilon} \quad$ Error rate $=\mathrm{d} \varepsilon / \mathrm{dt}$

$\ddot{\xi} \quad$ Error acceleration $=\mathrm{d}^{2} \varepsilon / \mathrm{dt}^{2}$

$\overline{e^{2}} \quad$ Mean-square error

$\sqrt{\epsilon^{2}} \quad$ Root-mean-square error

$\mathrm{F}(\mathrm{t}) \quad$ System weighting function $=\mathscr{L}^{-1} \mathrm{~F}(\mathrm{~s})$

$F_{0}(t) \quad$ Weighting function for elementary secondorder system (Fig. 3)

$F^{\prime}(t) \quad$ Weighting function for $F_{0}(t)$ system when using error-correction unit (ECU)

$F(s) \quad$ System transfer function $=E_{0}(s) / E_{i n}(s)$

$F_{0}(s) \quad$ System transfer function for elementary second-order system (Fig. 3)

$\mathrm{G}_{\mathrm{n}}(w) \quad$ Noise power spectral density

$\mathrm{G}_{0} \quad$ Power spectral density for "white" noise

$\gamma \quad$ Ratio, $\frac{\text { Magnitude of system output at } T_{S}}{\text { Value of input ramp } V T_{B}}$

i Electrical current

K S\%stem constant (Eq. 2)

k Power magnifier

L Electrical inductance (inertia)

$\mathrm{L}_{\mathrm{n}} \quad$ Sequential input program (Sec, VIIt. B)
Symbol

$M_{n} \quad$ Sequential input program (Sec. VIII. B)

P Step-positional input signal

p Real pole

$9 \mathrm{~m} \quad$ Electrical phase angle

$\varphi_{n}(\tau)$

Autocorrelation function for $T=0$. Also $\varphi(0)=\sigma^{2}$

In Ratio of nth positive or negative peak response to input step amplitude

R Electrical resistance

D Correctional step-input signal

$\dot{\rho}$

Sw

Correctional ramp-input signal

Analog switch

$\mathrm{S} / \mathrm{H} \quad$ Sample-hold device

$\mathrm{s}$

$1 / \mathrm{s}$

A zero at the origin

A pole at the origin $\}(\mathrm{Eq} .2)$

$\sigma_{0}$

$T_{1,2,3 \ldots}$

Standard deviation of system output error

Temperatures $1,2,3, \ldots$, etc.

$\mathrm{T}(\mathrm{t}) \quad$ Temperature as a function of time

$\mathrm{T}_{0}$

Initial-condition temperature

$\mathrm{T}_{\mathrm{s}}$

System setting time $=\pi / \omega$

Time

T System time constant $=$ RC

HK Microdegrees Kelvin (temperature)

W

Velocity input signal, i.e., ramp $=\mathrm{Vt}$

Wattage

We $_{\mathrm{e}} \quad$ Electrical wattage

$W_{\alpha} \quad$ Equivalent wattage due to alpha heating

Wo $_{0} \quad$ Empty-chamber wattage (calorimeter)

$w_{n}$

Undamped natural frequency $=1 / \sqrt{\mathrm{LC}}$ (Fig. 3)

z

Actual frequency $=\omega_{n} \sqrt{1-6^{2}}($ Fig. 3)

A real zero

Damping ratio: $\zeta=\alpha / \omega_{n}$ 


\author{
METHODS AND MEANS FOR \\ CONTROL OF ERROR IN \\ PRECISION INSTRUMENTS AND SYSTEMS
}

by

Robert N. Lewis

\title{
ABSTRACT
}

The rapid and precise response of analog measurement and actuating systems has become the limiting factor in instrumentation since the availability and use of computer-derived commands. The existence of thousands of analog devices with slow time response and dynamical errors in response gives rise to the need for simple programming methods to improve their performance.

This report is written for experimenters and practicing engineers who must, because of budget and/or time limitations, push their present system's performance to the limit. It can also aid in the design of new systems.

The method described consists of reducing a system's response characteristics to those of an elementary system, $F_{0}(s)$, and applying appropriate programming for positive control. With simple techniques, an order-of-magnitude improvement in response time and accuracy is, in many cases, achievable. Microprocessor or minicomputer control is possible and can be used to achieve fully adaptive systems having superior control characteristics. The performance of noiselimited systems can also be dramatically improved.

In addition, complex, time-varying systems can be computer-controlled by sequential programming, so as to be fully adaptive to changing conditions. With additional effort, the method may prove valuable in potentially dangerous sys tems checkout situations. For example, when faced with control problems during flight testing of a complex aircrift, application of the method may provide a degree of control beyond that normally available, such that safe landing of the experimental aircraft is possible. 


\section{INTRODUCTION}

The precise and rapid measurement and control of shaft positions, motor speeds, temperatures, voltage and current levels, etc., have become of critical importance since the availability and use of computer-derived commands. Accuracies in the part-per-million range are routinely desired and commaisded in modern research applications, and it is highly desirable in most instances that the commanded value be achieved in minimum time. Although the average budget-limited researcher might be abie to obtain a minicomputer or to time-share computer facilities, he must usually interface to some older analog equipment. Most of these suffer limited time response, as compared with the time spent in generating a command.

Faced with the requirements for improving response times and accuracies of existing instruments, the researcher may be severely limited in scope. For example, one of the few adjustments that can easily be made on closed-loop instruments is the loop gain. This can usually be adjusted with (a) a front or rear panel control, (b) an internal control, or (c) replacing a fixed resistance with a variable one to be located either on the front or rear panel. The effect of gain adjustment for a second-order system can be seen from its response to a step change. For a system with a transfer function of

$$
F(s)=\frac{A}{s^{2}+2(5) s+A},
$$

where $\mathrm{A}$ is the loop gain, the following table can be constructed.

\begin{tabular}{lcc}
$\frac{A}{50}$ & $\begin{array}{c}\text { Time to First } \\
\text { Maximum, Ts }\end{array}$ & $\begin{array}{c}\text { Percentage Overshoot at } \\
\text { First Maximum }\end{array}$ \\
\cline { 2 - 3 } 100 & 0.628 & 4.3 \\
200 & 0.363 & 17.0 \\
400 & 0.237 & 23.0 \\
& 0.162 & 43.0
\end{tabular}

According to this table, step response time to the first overshoot maximum is reduced significantly for an increase in gain. The problem is that the overshoot becomes large and the system "rings" in a damped mode for some time. The techniques and expertise required to yield both fast response and fast settling time are time-consuming to acquire and of little interest to the average scientist or practical engineer. Yet these are the people who most desperately require simple solutions.

This report describes a method that can yield a currect response at the first overshoot maximum and maintain it (i.e., no "ringing"). This is 
accomplished by arranging that for each input, an auxiliary input is applied so that the output due to the combined inputs is correct in the "minimum time" and remains so. ("Minimum time" is defined here as the time to the first overshoot maximum using maximum gain without undue oscillation.) The method allows for optimum response to a ramp input and to a parabolic input as well as optimum response to a step input when the system constants have So n determined. (Several methods for determining system constants are 4. (nribed.) Since any normal input can be described in terms of steps, ramps, itid parabolas, a system controlled by the methods described in this report will respond in an optimum manner to any normal input.

In addition, application of the methods presented can provide an immediate reduction in the probable error of a measurement due to the internal noise of the measuring instrument. It is shown in Sec. X that noise-generated errors in system response lead to a probable measurement error, $\sigma_{\bar{x}}$, that can be reduced by an increase in measurement time only to a limiting value. Use of the methods described here can reduce the probable error by at least a factor of five below that normally obtained.

The text has been substantially reduced in volume by placing derivation summaries and proofs in condensed form in the figures. Although this puts considerably more burden on the reader interested in mathematical details, it allows for a more coherent and simplified description of the method and its. applications.

\section{THE RESPONSE UF PHYSICAL SYSTEMS}

The transfer function of a linea $:$ constant-coefficient system is defined as the ratio of the Laplace-transformed output to the Laplace-transformed input:

$$
F(s)=\frac{E_{\mathrm{O}}(\mathrm{s})}{E_{\text {in }}(\mathrm{s})} .
$$

Since $F(s)$ is normally a rational function, it can be factored into linear and quadratic factors of seven basic forms: ${ }^{1-3}$

1. K, a system constant

2. s, a zero at the origin

3. $1 / \mathrm{s}$, a pole at the origin

4. $\frac{s+z}{z}$, a real zero 
5. $\frac{p}{s+p}$, a real pole

6. $\frac{s^{2}+2 \alpha s+\omega_{n}^{2}}{\omega_{n}^{2}}$, a pair of complex conjugate zeros

7. $\frac{\omega_{n}^{2}}{s^{2}+2 \alpha s+w_{n}^{2}}$, a pair of complex conjugate poles.

Thus the general form for Eq. 1 is

$$
F(s)=\frac{K \prod_{j=1}^{J}\left(s+z_{j}\right) \prod_{n=1}^{N}\left(s^{2}+2 \alpha_{n} s+a_{n_{n}}^{2}\right)}{\prod_{k=1}^{K}\left(s+p_{k}\right) \prod_{m=1}^{M}\left(s^{2}+2 \alpha_{m} s+x_{n m}^{2}\right)}
$$

The response of $F(s)$ to a unit step input is $F(s)$ s. The time respunse (if the re are no repeated roots) is

$E_{0}(t)=K\left[1+\sum_{k=1}^{K} A_{k} \exp \left(-P_{k} t\right)+\sum_{m=1}^{M} \frac{B_{m}}{\omega_{m}} \exp \left(-\alpha_{m} t\right) \sin \left(\omega_{m} t+\sigma_{m}\right]\right.$.

where $A_{k}$ and $B_{m}$ are ants that depend upon the poles and zeros of Eq. 2

The first term of Eq. 3 is the steady-state response, and the next two terms represent the trapisient error. The second term is the transient error due to the real poles of Eq. 2. The third term is the transient error due to the presence of zeros both real and complex and to the presence of complex conjugate poles. The complex roots $\alpha_{m}$ of the complex conjugate poles control the damping of the sinusoidal ofillations of frequency, $x_{m}$, the imaginary part of the roots of the complex conjugate poles. The zeros of the systom control the amplitudes (by modifying $\mathrm{B}_{\mathrm{m}}$ ) and the phases (by controlling $\mathrm{F}_{\mathrm{m}}$ ) of the damped oscillations. (For further explanation see apendix.)

Repeated real poles give rise to additional terms in Eq. 3. For a second-order real pole, the response is

$$
\mathscr{L}^{-1}\left\{\frac{p^{2}}{(s+p)^{2}}\right\}=e^{-p t}+p t e^{-p t}
$$

For a second-order pair of complex conjugate poles, the response is

$$
\mathscr{L}^{-1}\left\{\frac{\omega_{n}^{4}}{\left(s^{2}+2 \alpha s+\omega_{n}^{2}\right)^{2}}\right\}=\frac{\omega_{n}^{4}}{2 \omega^{3}} \mathrm{e}^{-\alpha t} \sin \omega t-\frac{\omega_{n}^{4}}{2 \alpha^{2}} t e^{-\alpha t} \cos x t .
$$


When poles and teros are close together in magnitude, they are termed dipoles. The zero tends to cancel the pole, and thas a dipole affects the system only slightly.

The response of $F(s)$ to a unit velocity input is $F(s) / s^{2}$. The time re sponse will have the same form as for Fi. 3, except that an additional summa tion of constants may appear. This term represents the steady-state error due to the $1 / \mathrm{s}^{2}$ inpout.

The response of $F(s)$ te a unit-acceleration input is $F(s) / s^{3}$. The time response will have the same form as for Eq. 3. except that both the summation of constants and an additional summation of constants that a re multiplied with tame may appear. These terms represent the steady-state error due to the 1 s $^{3}$ input.

The steady-state errors due to velocity and acceleration inputs can be described in terms of pole and zero locations

The nature of the response of systems can thus be summarized as follows

1. Poles on the aegative real axis give rise to terms of the ferm $e^{-a t}$, te-at, etc... depending on the order of the pole.

2. Complex poles in the left half plane give rise to damped sine waves, i.e.., terms of the form ke ${ }^{-a t} \sin a t, k t e^{-a t} \cos \omega t$, etc., depending on the order of the pole.

3. Zeros of the transform contribute to amplitudes and phase angles, but do not change the form of the time function.

4. Dipoles affect the response only slightly and may be largely dis regarded.

5. Steady-state errors appear for inputs of $1 / \mathrm{s}^{2}$ and $1 / \mathrm{s}^{3}$ due to pole and zero locations.

\section{ERROR IN RESPONSE}

According to Eq. 1, the output, $E_{o}(s)$, due to an input, $E_{\text {in }}(s)$, is

$$
\mathrm{E}_{\mathrm{o}}(\mathrm{s})=\mathrm{E}_{\mathrm{in}}(\mathrm{s}) \mathrm{F}(\mathrm{s})
$$

Ideally the output will be the input amplified by a constant; i.e.,

$$
E_{o}(s)(\text { ideal })=\mathrm{KE}_{\text {in }}(\mathrm{s}) \text {, }
$$

where $\mathrm{K}$ is the system gain constant as defined in Eq. 1. 
The output error, e(s), is defined as

Error = desired output - actual output;

$$
\epsilon(s)=K E_{\text {in }}(s)-F(s) E_{\text {in }}(s)
$$

The transfer function $F(s)$ can also be written as

$$
F(s)=K F(1)(s) \text {, }
$$

where $F(1)(s)$ signifies a normalized unity-gain function; i.e.,

$$
F(1)(s)=F(s) / K \text {. }
$$

Using Eq. 9 in Eq. 8 provides

$$
\epsilon(s)=K_{\text {in }}(s)[1-F(1)(s)] \text {. }
$$

Equation 10 accounts for transient as well as steady-state errors.

\section{A METHOD FOR ERROR REDUCTION}

A method for reducing the output error of a system is to arrange that for each input, $E_{1}(s)$, an auxiliary input, $p_{1}(s)$, is applied so that the combined outputs from $E_{1}(s)$ and $p_{1}(s)$ most nearly correspond to the desired output $\mathrm{KE}_{1}(\mathrm{~s})$. The ideal value for $\rho_{1}(\mathrm{~s})$ can be determined by writing

$$
\left[E_{1}(s)+\rho_{1}(s)\right] F(s)=K_{1}(s)
$$

By use of Eq. 9, the solution $\mathrm{fcr}_{\mathrm{O}_{1}}(\mathrm{~s})$ is

$$
\rho_{1}(s)=E_{1}(s) \frac{1-F(1)(s)}{F(1)(s)}
$$

The scheme might be implemented as shown in Fig. 1. For example, suppose the system, $F(s)$, is a tape-controlled table that translates a piece pari in $x$ and $y$ for drilling of holes according to the program $E(s)$. The dynamic response of the table may be such that relatively long waiting periods may be required after application of a signal until all transient errors are reduced sufficiently close to zero. If an additional input o(s) is made simultaneously with $E(s)$, where $p(s)$ is obtained by use of Eq. 12, the transient errors will be reduced to zero much more rapidly, and significantly greater shop production will be possible. 


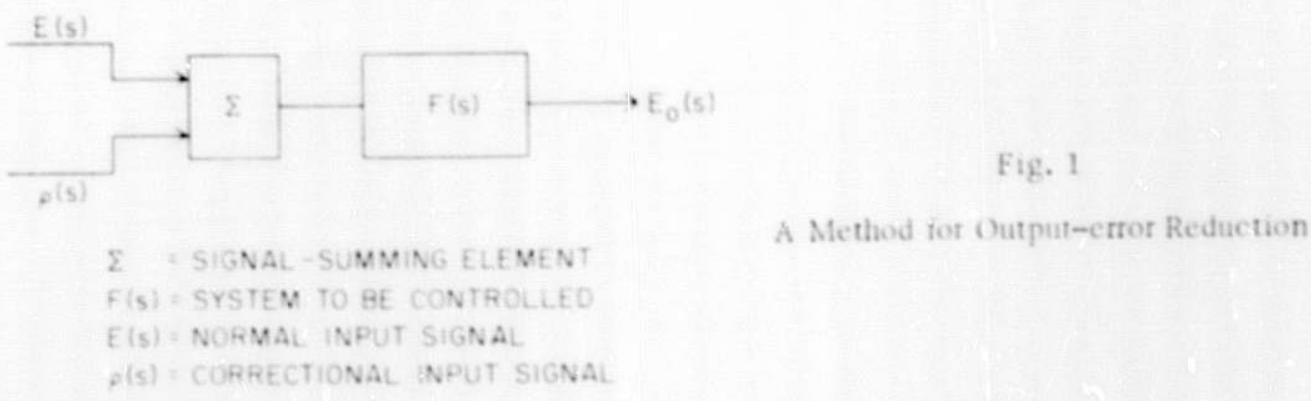

V. APPI.CATION TO AN ELEMENTARY POSITIONAL SYSTEM

A. Open-loop Error Correction

Examples of this application are:

1. Periodic driving functions; for example, a triangular waveform to drive a scanning mirror in an optical scanning system.

2. Step-positional inputs; for example, a magnetic field that is stepchanged in amplitude for isotopic ratio analysis in a mass spectrometer. Another example is a tape-controlled machine that translates a piece part in $x$ and $y$ for drilling of holes.

The method is implemented as follows

1. - The error-correction device is inserted (at point X) between the input signal and the system to be controlled. (See Fig. 2.)

2. The error-correction device consists of a summing amplifier with several inputs. There are several error-correction signals, and one input is the normal input signal. Thus, if no error-correction signals are generated, the system performs normally.

3. A few simple tests and adjustments are performed.

4. The normal input signal is applied. Auxiliary signals are generated by the error-correction device and applied to the summing amplifier. Their intended magnitudes and waveforms (steps, ramps, and exponentials) are such that, at the end of a time interval $\mathrm{T}_{\mathrm{s}}$, the output error is zero, its time-rate-of-change is zero, and no erroneous energy is stored in any of the internal components of the system. Thus the system output error remains at zero for $t \geq T_{s}$. 


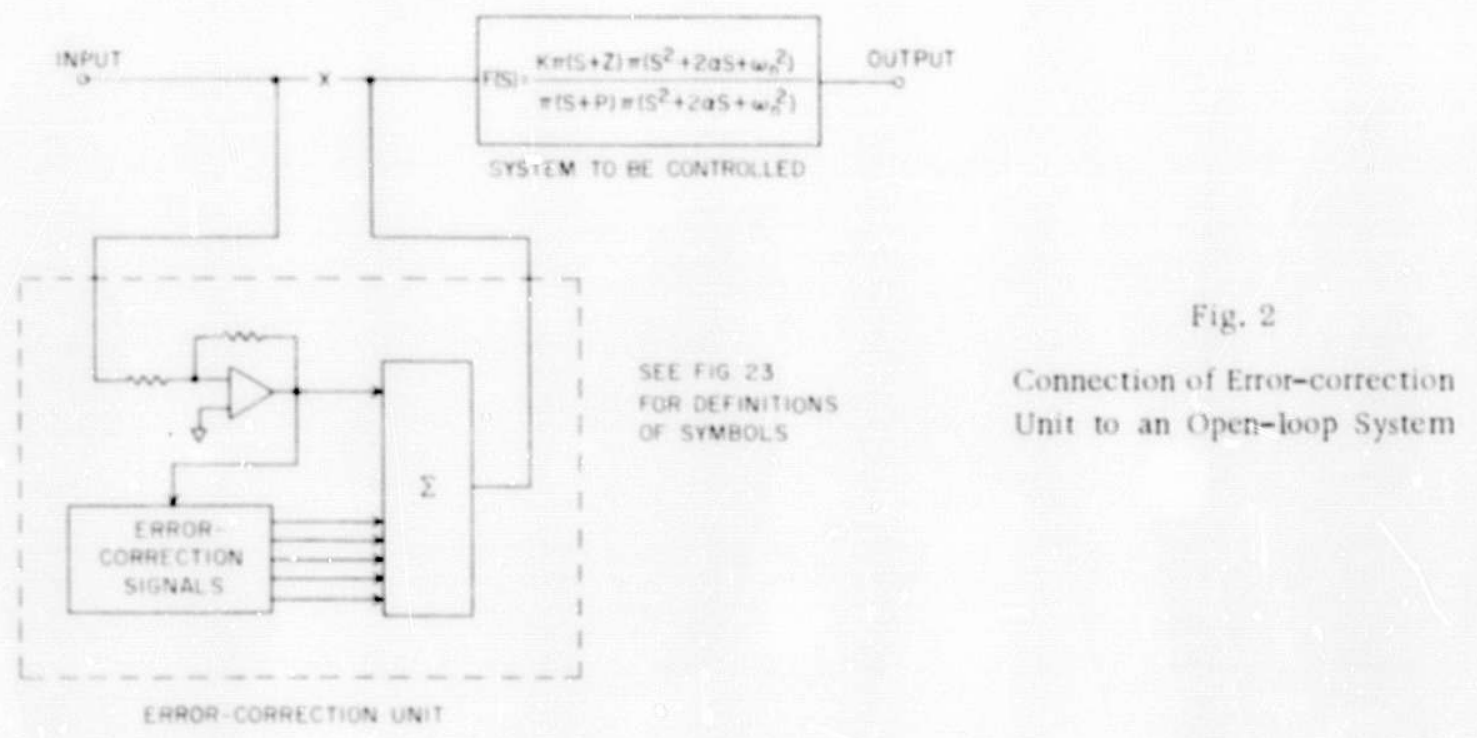

1. Application of the Method for Error Reduction

The elementary underdamped positional system has two complex conjugate poles and no zeros. For unity gain, it has the transfer function

$$
F_{0}(s)=\frac{w_{n}^{2}}{s^{2}+2 \alpha s+w_{n}^{2}} .
$$

The electrical analog is shown in Fig. 3 with the mathematical relationships defined. Mechanical analogs can be shown in which inductance is related to inertia, capacitance to spring compression, and resistance to frictional losses. The time response of the system to delta, step, velocity, and acceleration inputs is shown in Fig. 4.

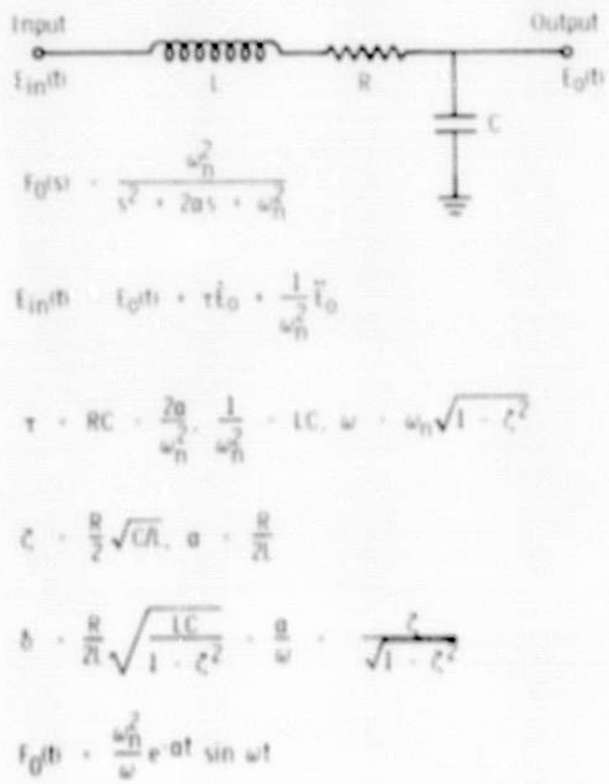

Fig, 3. Electrical Cireuit Having Characteristies of Elementary Positional System

$$
\begin{aligned}
& \text { Input Arsponse } \\
& \text { Lo. } \quad 2^{-28 r_{0} s u}=8 \frac{u^{2}}{u} e^{-a t} \sin u t
\end{aligned}
$$

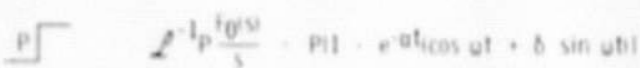

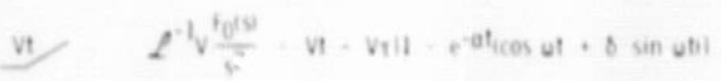

$$
\begin{aligned}
& \frac{v}{v_{n}} \frac{u_{n}^{2}}{v} e-a t \sin u t
\end{aligned}
$$

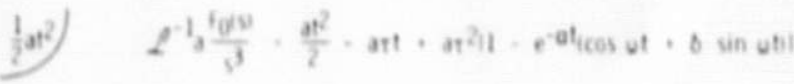

$$
\begin{aligned}
& \frac{a t}{v_{n}} \frac{v_{n}^{2}}{v} e-a t \sin u t \\
& \sum_{n}^{3} n+e^{-a t i c o s} u t \cdot 8 \sin u t s t
\end{aligned}
$$

Fig. 4. Time Response of $\mathrm{F}_{0}(\mathrm{~s})$ System to Delta, Step, Velocity, and Acceleration Inputs 
Applying Eq. 12 to the $F_{0}(s)$ system yields

$$
\rho(s)=E(s) \frac{s^{2}+2 \alpha s}{\omega_{n}^{2}} .
$$

With the relations between $\omega_{n}, \alpha$, and $\tau$ shown in Fig. 3, Eq. 14 becomes

$$
\rho(s)=s \tau E(s) \frac{s+2 \alpha}{2 \alpha}
$$

Figure 5 shows a system for generating the correctional input, $p(s)$, as required by Eq. 15.

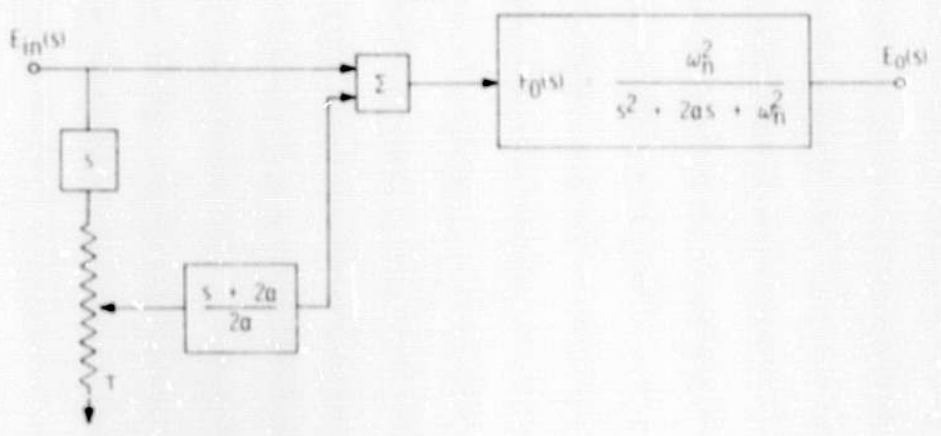

\section{Fig. 5}

Method for Providing Correctional Input, $\rho(s)$, for the $F_{0}(s)$ System

As a test of the scheme, assume an input signal $E_{\text {in }}(t)=\frac{1}{2} a t^{2}$ $\left[E(s)=a / s^{3}\right]$. Figure 6 indicates that there is considerable output error. According to Eq. 14, the correctional input should be

$$
\begin{aligned}
& \rho(s)=\frac{a}{s^{3}} \cdot \frac{s^{2}+2 \alpha s}{\omega_{n}^{2}}=\frac{1}{s} \frac{a}{\omega_{n}^{2}}+\frac{a}{s^{2}} \tau, \\
& \text { Input Signal }
\end{aligned}
$$

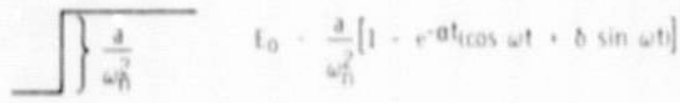

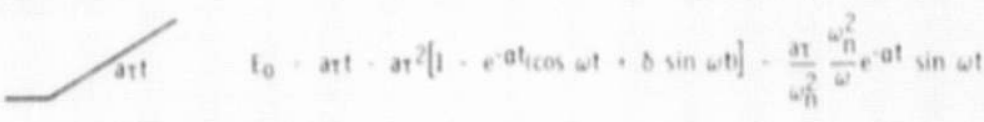

$$
\begin{aligned}
& \overline{\frac{2 \text { inputs }}{3 a t^{2}+a t+\frac{3}{\sigma_{n}^{2}}}} \frac{2 \text { Outputs }}{\text { lat }^{2}}
\end{aligned}
$$

Fig. 6. Response of $\mathrm{F}_{0}(\mathrm{~s})$ System to an Input Signal E(t) $+\rho(t) \quad \frac{1}{a} t^{2}+a r t+a / w \frac{2}{n}$ 
that is, an input ramp and an input step function. These two inputs are also shown in Fig. 6, with the resultant output. The output is seen to exactly equal the input when the two additional inputs are applied along with the normal input. For a ramp input signal $\left(=\mathrm{V} / \mathrm{s}^{2}\right)$, the required correctional signals are a step function equal to $V \tau$ and a delta function whose area is $V / \omega_{n}^{2}$. This is shown in Fig. 7, which shows the output to exactly follow the input when the correctional signals are also applied.

Input Signa

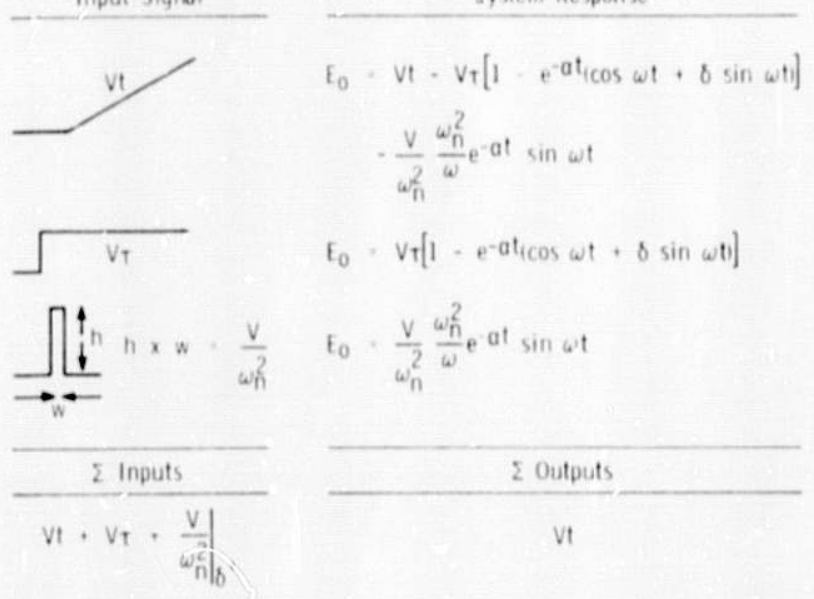

Fig. 7. Response of $F_{0}(s)$ System to an Input Signal $E(t)+\rho(t)=V t+V \tau+V /\left.\omega_{n}^{2}\right|_{\delta+}$
For a step-function input, $\mathrm{P} / \mathrm{s}$, the theory demands correctional signals $\mathrm{sP} / \omega_{n}^{2}$ and $\tau \mathrm{P}$. That is, it demands a delta function $\tau P$ and the first timederivative of a delta function $\mathrm{sP} / \omega_{\mathrm{n}}^{2}$. Theoretically, the three inputs $\mathrm{P} / \mathrm{s}$, $\mathrm{sP} / \omega_{\mathrm{n}}^{2}$, and $\tau \mathrm{P}$ would cause the output to correctly respond to $\mathrm{P} / \mathrm{s}$. This is physically impossible, however, since infinite slew rates would be required.

Because it is physically im possible for the output of a secendorder system to change position in zero time, the scheme will be to allow a settling time, $T_{S}$, for the change to occur and to demand that the output be errcr-free after this time. The time $\mathrm{T}_{\mathrm{S}}$ choser is one-half period of the damped ringing frequency. Since time intervals between successive positive and negative peaks of the step response of the $F_{0}(s)$ system are equal, the time $\mathrm{T}_{\mathrm{S}}$ h re is the time between application of a step and the time of the first positive peak of the response.

The first derivative of the time response to a step input $\mathrm{P}$ is $\mathrm{P}\left(\omega_{n}^{2} / \omega\right) \mathrm{e}^{-\alpha t} \sin \omega t$. By equating this to zero, we find the times for occurrence of the positive and negative peaks of the response to be $\pi / \omega, 2 \pi / \omega, \ldots, n_{\pi} / \omega$. The output, $\psi_{n}$, at these times can be calculated as

$$
\psi_{n}=P \frac{\omega_{n}^{2}}{\omega} \int_{0}^{n \pi / \omega} e^{-n \alpha t} \sin n \omega t d t=P\left[1-(-1)^{n} e^{-n \alpha \pi / \omega}\right] .
$$

Upon application of the step, the initial error, $\epsilon_{0}$, is $\epsilon_{0}=P$. The error at $\mathrm{t}=\pi / \omega$ is $\varepsilon_{1}=\mathrm{P}\left(1-\psi_{1}\right)=\epsilon_{0}\left(1-\psi_{1}\right)$. The error at $\mathrm{t}=2 \pi / \omega$ is $\varepsilon_{2}=\epsilon_{0}\left(1-\psi_{2}\right)$, etc. Using Eq. 17, we can show that $1-\psi_{2}=\left(1-\psi_{1}\right)^{2}, 1-\psi_{3}=\left(1-\psi_{1}\right)^{3}$, and that, in general, 


$$
\epsilon_{\mathrm{n}}=\epsilon_{0}\left(1-\psi_{i}\right)^{\mathrm{n}},
$$

which yields the ratio

$$
\frac{\epsilon_{\mathrm{n}+1}}{\epsilon_{\mathrm{n}}}=1-\psi_{1}
$$

The error rate, $\dot{\epsilon}$, can be compared at a time $\pi / \omega$ in the future with the present rate; i.e.,

$$
\frac{\dot{\epsilon}(t+\pi / \omega)}{\dot{\epsilon}(t)}=\frac{P \frac{\omega_{n}^{2}}{\omega} \exp [-\alpha(\pi / \omega+t)] \sin \omega(\pi / \omega+t)}{P \frac{\omega_{n}^{2}}{\omega} e^{-\alpha t} \sin \omega t}=1-\psi_{1} .
$$

Thus,

$$
\frac{\dot{\varepsilon}_{\mathrm{n}+1}}{\dot{\varepsilon}_{\mathrm{n}}}=1-\psi_{1} \text {. }
$$

By the same process, it can be shown that

$$
\frac{\ddot{\epsilon}_{\mathrm{n}+1}}{\ddot{\epsilon}_{\mathrm{n}}}=1-\psi_{1} \text {. }
$$

The general requirement for the input values of a unit step are shown in Fig. 8a. For simplicity, we will use the weighting function $F(t)$ or $\mathrm{F}(\mathrm{s})$ and delta functions, since the step response is the time integral of the weighting function. At $t=0$, a value $]-\rho$ is applied. At $t=T_{S}$, a second value $p$ is applied. The requirement is that the response for $t z T_{S}$ be zero. Figure $8 \mathrm{a}$ shows a theoretical value for $\rho$ for accomplishing this.

$$
\begin{aligned}
& 1 \text { - pil at General Requirement } \\
& \text { (1- pifisiexph-sts }) \text { pfis) }=0 \\
& \text { p. } \frac{f(s i e x p(-s / s)}{F(s)-F(s) e x p\left(-s T_{s}\right)} \\
& \text { b) Test for foiti System } \\
& f_{0} t t \cdot \frac{w_{n}^{2}}{\omega} e^{-a t} \sin \omega t \\
& \text { Is. } \pi / 6 \\
& \text { p. } \frac{\frac{\omega_{n}^{2}}{\omega} e^{-a t e}-a \pi / \omega \sin \omega t}{\frac{\omega_{n}^{2}}{\omega} e^{-a t} \sin \omega t \cdot \frac{\omega_{n}^{2}}{\omega} e^{-a t} e^{-a \pi / \omega} \sin \omega t} \\
& \frac{e^{-0 \pi / \omega}}{1 \cdot e^{-0 \pi / \omega}} \cdot \frac{\phi_{1}-1}{\theta_{1}}
\end{aligned}
$$

Fig. 8

General Requirement for Smooth Stepfunction Response in Time Interval $\mathrm{T}_{\mathrm{s}}$ 
Shown in Fig. $8 \mathrm{~b}$ is the solution of $\rho$ for the $F_{0}(\mathrm{~s})$ system. It shows that a value of $\left(\psi_{1}-1\right) / \psi_{1}$ is satisfactory. It will become apparent later that the $F_{0}(\mathrm{~s})$ system is unique in accommodating such a simple program. This is true because of its dynamical symmetry described by Eqs. 19, 21, and 22 and because $\mathrm{T}_{\mathrm{S}}(2)=2 \mathrm{~T}_{\mathrm{S}}(1)$, etc.

Because of the dynamic symmetry of the $F_{0}(s)$ system, the program shown in Fig. 9 is applicable for step-positional control. For a desired change in position, $P$, an input of $P(1-\rho)=P / \psi_{1}$ is applied for a time interval $\pi / \omega$. At this instant, the input is increased to the value $P$. The output rises smoothly to a value $\mathrm{P}$ at $\mathrm{t}=\pi / \omega$ and remains at that value thereafter. The device shown in Fig. 5 can be further improved as shown in Fig. 10, so that step inputs are accommodated. Step inputs cause a large output from the differentiator, thus tripping the monostable element and subtracting the fraction $(\psi-1) / \psi$ from the input step for the time period $\pi / \omega$. During this period, the output of the $(s+2 \alpha) / 2 \alpha$ element is gated-off. The time constant $\mathrm{R}_{1} \mathrm{C}_{1}$ (which is short in comparison with the time period $\pi / \omega)$ references the input of amplifier $A_{1}$ to the dc level of the input signal. Thus, only the step-level change is operated upon.

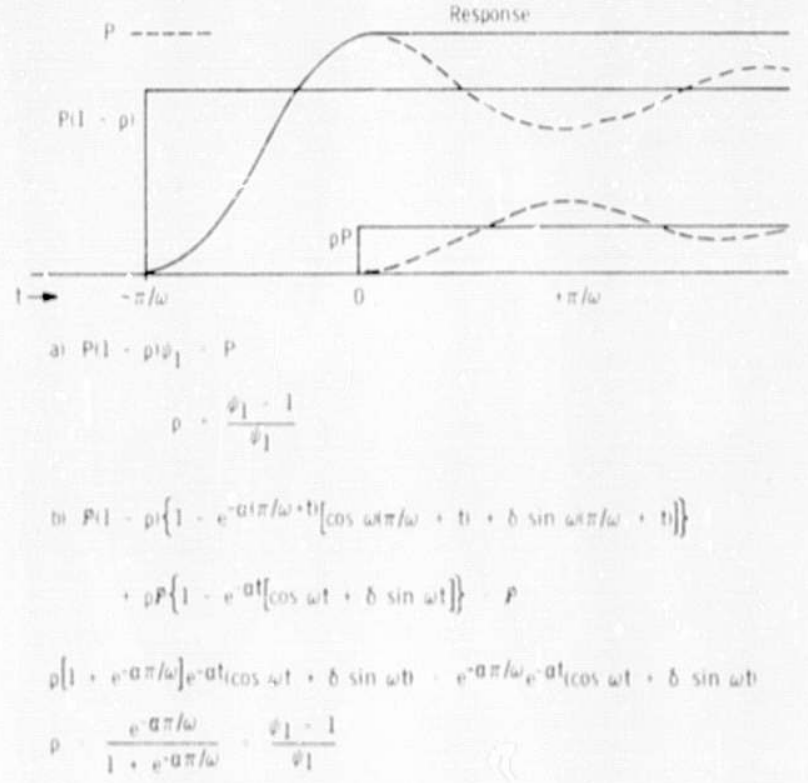

Fig. 9. Input Program for Step-positional Respoise

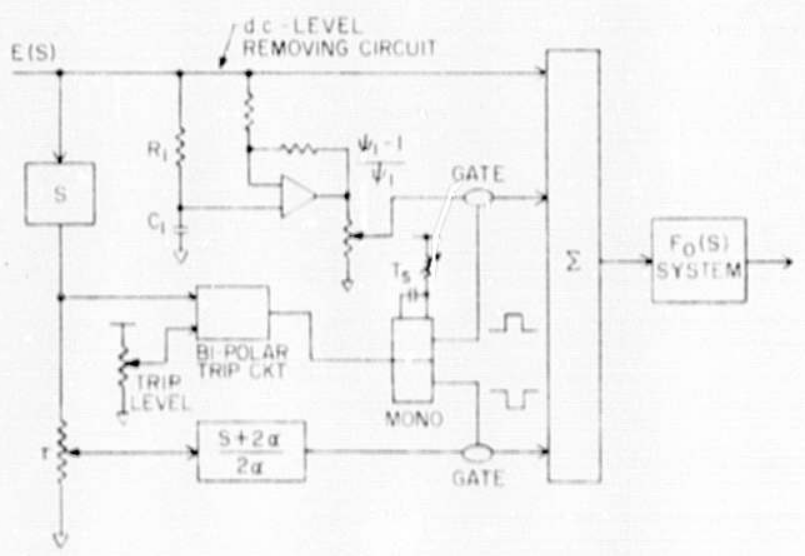

Fig. 10. Control Device for Improved Open-loop Control of $\mathrm{F}_{0}(\mathrm{~s})$ System

2. Method for Improving Response Time

Most $F_{0}(s)$ systems are closed-loop systems. Such is the case, for example, for the simple positional servo. Changing loop gain changes the dynamical response of the system because loop gain affects the "spring" of a mechanical system whose electrical analog is $1 / \mathrm{C}$, the reciprocal of capacitance. We can thus write (referring to Fig. 3) 


$$
\begin{aligned}
& \frac{1}{C} \simeq \mathrm{A}, \\
& \omega_{n}=\frac{1}{\sqrt{L C}}=k_{1} \sqrt{A},
\end{aligned}
$$

and

$$
\zeta=\frac{R}{2} \sqrt{C / L}=k_{2} / \sqrt{A} .
$$

It is seen that the product $\zeta \omega_{n}$ is not affected by gain changes. Thus the trans fer function can be written

$$
F_{0}(s)=\frac{k_{1}^{2} A}{s^{2}+2 k_{1} k_{2} s+k_{1}^{2} A} \text {. }
$$

For $k_{1}=i$,

$$
\Phi_{0}(s)=\frac{A}{s^{2}+2 k_{2} s+A} \text {. }
$$

As an example, we will assume a large mechanical system and a value of 5 for $k_{2}$. Table $I$ then results for changes in loop gain.

\begin{tabular}{cccccc}
\multicolumn{2}{c}{ TABLE I. Unit-step Response for $\mathrm{F}_{0}(\mathrm{~s})=\mathrm{A} /\left(\mathrm{s}^{\prime}+10 \mathrm{~s}+\mathrm{A}\right)$} \\
$\mathrm{A}$ & $\omega_{\mathrm{n}}$ & 5 & $\omega$ & $\begin{array}{c}\mathrm{T}_{\mathrm{s}}=\pi / \omega, \\
\mathrm{ms}\end{array}$ & $\begin{array}{c}\text { Percent } \\
\text { Overshoot }\end{array}$ \\
\hline 25 & 5.00 & 1.0 & - & - & - \\
50 & 7.07 & 0.707 & 5.00 & 628 & 4.3 \\
100 & 10.00 & 0.5 & 8.66 & 363 & 17.0 \\
200 & 13.23 & 0.378 & 12.25 & 237 & 23.0 \\
400 & 20.00 & 0.25 & 19.36 & 162 & 43.0 \\
1600 & 40.00 & 0.125 & 39.69 & 79 & 65.0 \\
\hline
\end{tabular}

Changing loop gain from 50 to 200 improves response time by a factor of 2.65 . Increasing the gain to 400 from 50 improves response time by a factor of 3.88 . The overshoots of 23 or $43 \%$ provide $\psi_{1}$ values of 1.23 and 1.43 , respectively, and these along with the values for $\mathrm{T}_{\mathrm{s}}$ of 0.237 or $0.162 \mathrm{~s}$ allow mechanization of the present control t'ieory. 


\section{Significant Overall Improvement in System Performance}

Consider an application for the system described in Table I, where the required positional error for a step change in position is $50.1 \%$. Figure 11 a shows the system response for various loop gains. Analysis will reveal that, for any loop gain, the settling time to $0.1 \%$ error is $>1.5 \mathrm{~s}$. By increasing the loop gain to 400 and applying the subject control method, we can achieve the dramatically improved performance of Fig. $11 \mathrm{~b}$. Here, settling to zero error is accomplished in $0.162 \mathrm{~s}$, an improvement in performance by at least an order of magnitude. Higher values for loop gain can lead to even shorter settling times.

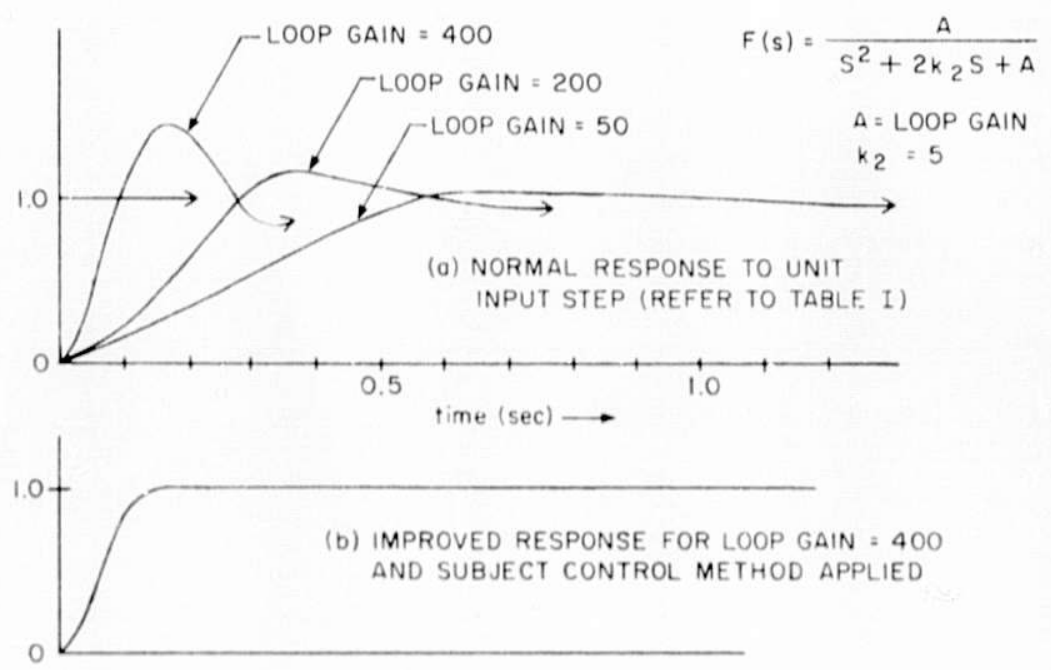

Fig. 11. Improvement of System Performance

For an existing large installation, where an order-of-magnitude improvement in performance is required, the experimenter must consider replacement of the system by a new system. If the new system design is based upon conventionally accepted methods, the servo motors and their drive amplifiers must have considerably higher power ratings and the feedback transducers must usually be of higher quality for improved resolution and accuracy. Purchase of the new system will probably involve a considerable expenditure of project funds.

A possible alternative approach is to install improved feedback transducers (if found to be necessary), to increase loop gain (if existing system components allow), and to apply the control method described here. This approach would have at least two advantages:

a. The experiment could begin at an earlier date because modification would require less ime than that for new system procurement.

b. The cost to the project would be significantly reduced. 
B. Closed-loop Error Correction

The error-correcting device can be connected in the feedback signal path of a closed-loop system. There are thousands of potential applications. Two examples are:

1. A regulated high-voltage power supply, in which occasional arcing interrupts data collection. The error corrector will return the output voltage to its correct value in minimum time, and with no overshoot that might normally lead to a new arc.

2. An autopilot controlled aircraft in level cruise subjected to occasional atmospheric turbulence. The error-correction mechanism will cause the aircraft to be returned to normal flight attitude in a smooth and rapid manner.

The method is implemented as follows:

1. The signal path is opened at any point in the system where the signal level is in the proper magnitude range (usually $1-10 \mathrm{~V}$ peak) and where

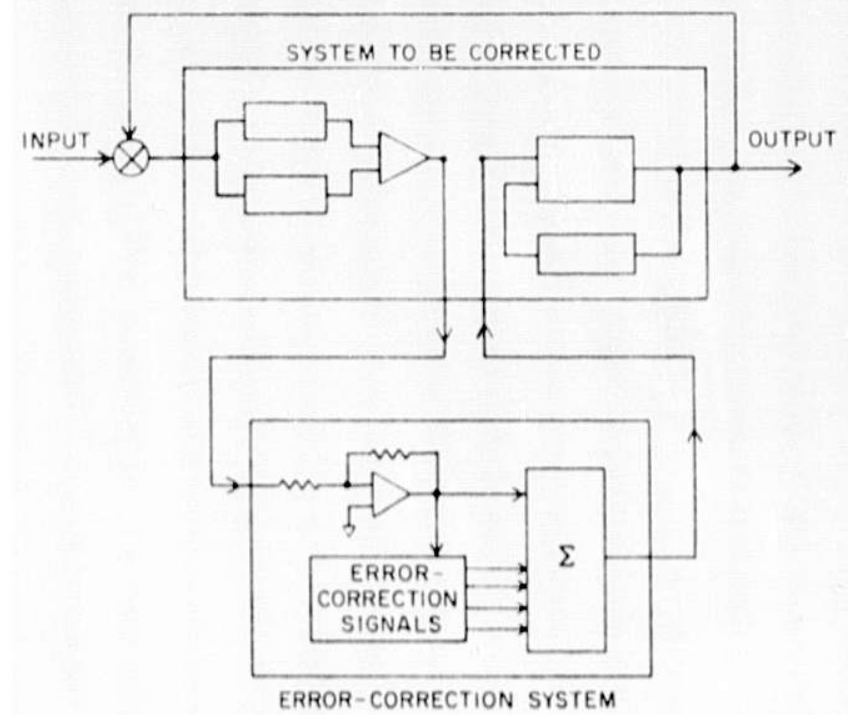

Fig. 12. Connection of Error-correction System to a Closed-loop System this point is the total signal and not a branch leading to a summing point. (See Fig. 12.) The transfer function is the same from any such point in the system back to the same point, regardless of its location. The errorcorrecting system (described in Sec. B. 3 below) is inserted at this point.

2. The error-correcting system consists of a summing amplifier with one input being the normal feedback error signal for this point. Thus, if no error-correcting signals are generated, the system will perform normally.

3. A few simple tests and adjustments are performed. These determine the values of certain fundamental parameters of the system to be controlled. These quantities, when multiplied by the present error and its time-rate-of-change, allow prediction of future errors and their time-rates-of-change for discrete time intervals, $T_{S}$, in the future. The time interval, $\mathrm{T}_{\mathrm{S}}$, is the allowed total settling time to zero for all future times of an existing positional error. It is also equal to $\pi / \omega$ where $\omega$ is the dominant frequency of the system. 
4. Auxiliary signals, consisting of voltage steps and tims iamps, are generated and applied to the summing amplifier. Thus they are in parallel with the normal feedback error signal and are not changed in magnitude (except in special cases) during the time interval $\mathrm{T}_{\mathrm{S}}$. Their intended magnitudes are such that, at the end of the time interval $T_{S}$, the error is zero and its timerate-of..change is zero. Thus, if no additional inputs to the primary system have been applied during $\mathrm{T}_{\mathrm{S}}$, there is no stored energy in the system, and if the error-correcting signals are removed at $\mathrm{T}_{\mathbf{S}}$, the error remains at zero.

5. In practice, the error is sampled again at $\mathrm{T}_{\mathbf{S}}(1)$, again at $\mathrm{T}_{\mathbf{S}}(2)$, etc. This allows for correction for new inputs as well as for further correc-

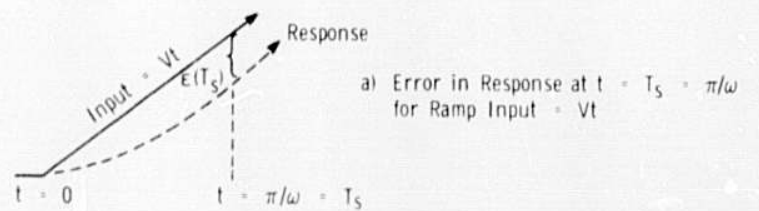

$E_{0}(t)=V t-V_{T}\left[1-e^{-a t}(\cos \omega t+\delta \sin \omega t)-\frac{V}{\omega_{n}^{2}} \frac{\omega_{n}^{2}}{\omega} e^{-a t} \sin \omega t\right]$

$E_{0}\left(T_{S}\right)=V T_{S} r=V T_{S} \cdot V_{T} \psi_{1}$

$\varepsilon\left(I_{S}\right) \cdot V T_{S}-E_{0}\left(T_{S}\right)=V_{T} \psi_{1}=V T_{S}(1-\gamma), \gamma=1-\frac{T \psi_{1}}{T_{S}}$

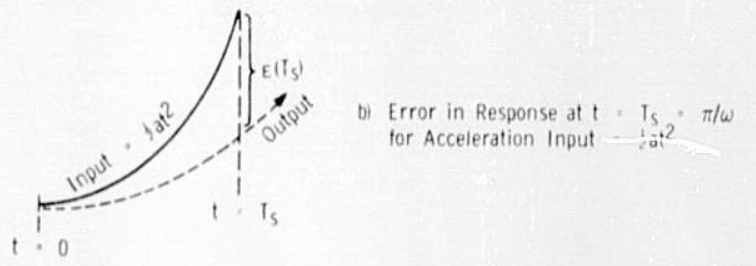

$$
\begin{aligned}
& E_{0}(t) \cdot \frac{a t^{2}}{2}=a t t+a T^{2}\left[1-e^{-a t}(\cos \omega t+\delta \sin \omega t)\right] \\
& +\frac{a \tau}{\omega_{n}^{2}} \frac{\omega_{n}^{2}}{\omega} e^{-a t} \sin \omega t-\frac{d}{\omega_{n}^{2}}\left[1-e^{-a t} i \cos \omega t+\delta \sin \omega t\right] \\
& E_{0}\left(T_{s}\right)=\frac{a T_{s}^{2}}{2} \beta=\frac{a T_{s}^{2}}{2}=a T_{s}+a T^{2} \phi \cdot \frac{a \psi_{1}}{\omega_{n}^{2}} \\
& E\left(T_{S}\right)=\frac{a T_{S}^{2}}{2} \cdot E_{0}\left(T_{S}\right)=a\left[T_{L}\left(T_{S}-T \psi+\frac{\psi}{\omega_{n}^{2}}\right]=\frac{a T_{S}^{2}}{2}[1-\beta]\right.
\end{aligned}
$$

Fig. 13. Definition of Additional Factors $\gamma$ and $\beta$ tion of any remaining error due to incorrectly measured or time-variable system parameters.

\section{Some Additional Factors}

We have above defined $\mathrm{P} \psi_{1}$ as the value of the output at $T_{S}(1)$ due to a step input of magnitude $\mathrm{P}$. With $\mathrm{T}_{\mathrm{S}}(\mathrm{l})$ thus known, if a ramp input $\mathrm{Vt}$ is applied, the value of the output at $T_{S}$ is not $V T_{S}$ but usually sone smailer value $V^{-} T_{5}$, as shown in Fig. 13a. We have

$$
\frac{\text { Magnitude of output at } \mathrm{T}_{\mathrm{S}}}{\text { Ramp value at } \mathrm{T}_{\mathrm{S}}}=\frac{\gamma \mathrm{VT}_{\mathrm{S}}}{\mathrm{VT}_{\mathrm{S}}}=\gamma \text {. }
$$

In a similar manner, the response to a parabolic input $=\frac{1}{2} a t^{2}$ can be measured at $T_{S}$ and the ratio of output to input calculated as

$$
\frac{\text { Output at } \mathrm{T}_{\mathrm{S}}}{\text { Input at } \mathrm{T}_{\mathrm{S}}}=\beta \text {. }
$$

This is shown in Fig. 13b.

\section{Positional Control}

Because the error $\varepsilon_{1}$ at $T_{\mathbf{S}}(1)$ due to an initial error $\epsilon_{0}$ is $\varepsilon_{0}\left(1-\psi_{1}\right)$, the required additional input, $p_{1}$, at $t=0$ to reduce the error to zero at $\mathrm{T}_{\mathbf{S}}(1)$ is (see Fig. 14)

$$
\psi_{1} \rho_{1}=-\epsilon_{0}\left(1-\psi_{1}\right) \text { or } \rho_{1}=-\epsilon_{0} \frac{1-\psi_{1}}{\psi_{1}} \text {. }
$$




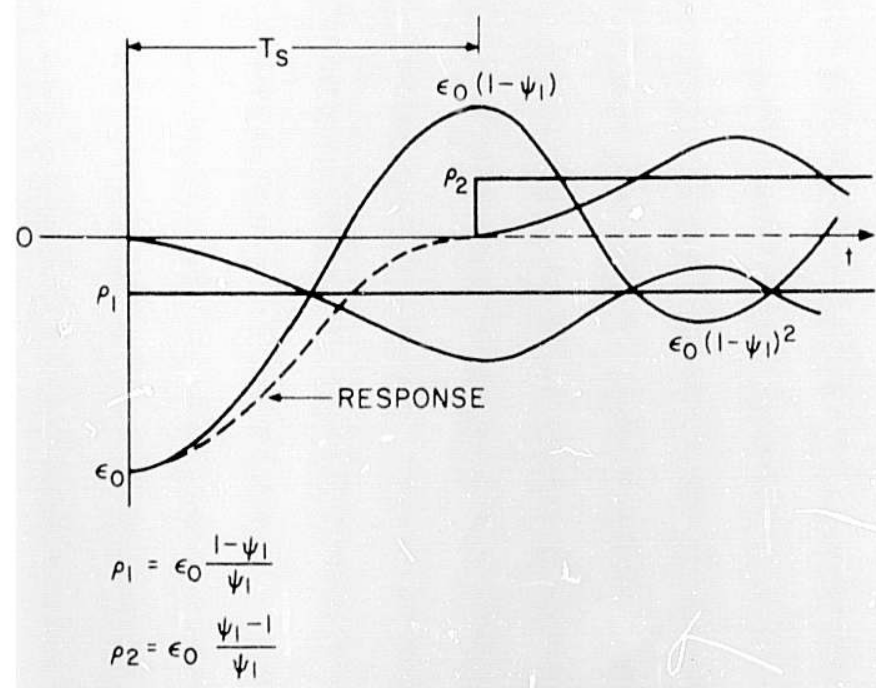

Fig. 14. Positional Error Control for Elementary System
This follows because the response to $\rho_{1}$ at $T_{S}(1)$ will be $\psi_{1} \rho_{1}=$ $-\epsilon_{0}\left(1-\psi_{1}\right)$, and this will cancel the error. At $T_{S}$, a second input, equal and opposite to $p_{1}$, is inserted:

$$
\rho_{2}=-\rho_{1}=\epsilon_{0} \frac{1-\psi_{1}}{\psi_{1}}
$$

Tine system response will be as shown by the dashed curve, reaching zero at $T_{s}$ and remaining at zero thereafter because the sum of the three responses is zero for $t \geq T_{S}$.

\section{Actual programming of} the correction signal will consist of applying $\rho_{1}$ at $t=0$ and removing it at $\mathrm{t}=\mathrm{T}_{\mathrm{s}}$. This is shown diagrammatically in Fig. 15.

Fig. 15

Progıamming of Positional Error Correction for Elemrntary System

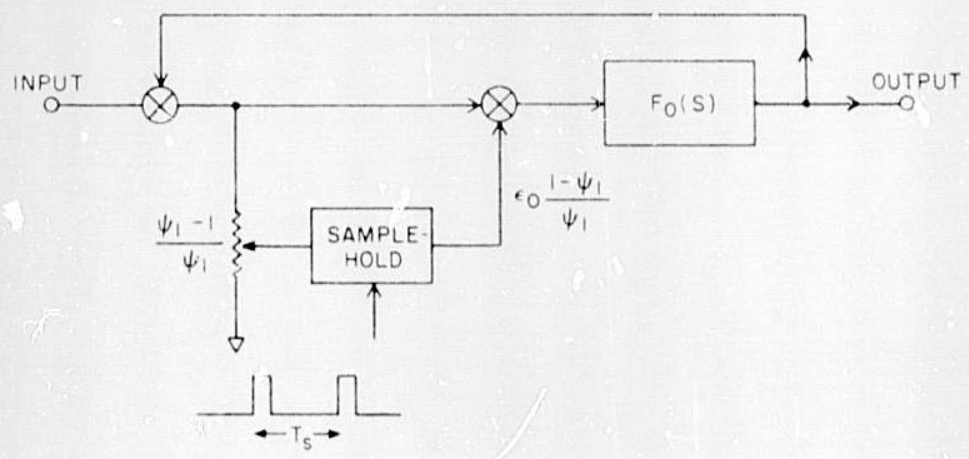

An error, when first observed, may have both a magnitude and a rate. In particular, this is trie when a system first comes out of saturation due to a large signal. In this case, it is desired to inject a signal set that will reduce both the error magnitude and the error rate to zero in a time $T_{S}$ after the first observation.

The situation is illustrated in Fig. 16. At $t_{0}$, the error set is $\varepsilon_{0}$ and $\dot{\epsilon}_{0}$. Because of dynamic symmetry, we can predict the set to have the values at $t_{0}+T_{S}$ of $\epsilon_{0}\left(1-\psi_{1}\right)$ and $\dot{\varepsilon}_{0}\left(1-\psi_{1}\right)$. To reduce the error rate to zero at $t_{0}+T_{S}$, a ramp, $\dot{p} t$, is applied of such magnitude that

$$
\dot{\rho} \psi_{1}=-\dot{\epsilon}_{0}\left(1-\psi_{1}\right) \text {, }
$$

or

$$
\dot{\rho}=-\dot{\epsilon}_{0} \frac{1-\psi_{1}}{\psi_{1}} \text {. }
$$


This ensures that the rate response to the ramp at $t_{0}+T_{S}$ is equal and opposite to the rate error at $t_{0}+T_{S}$, and thus the output rate is zero.

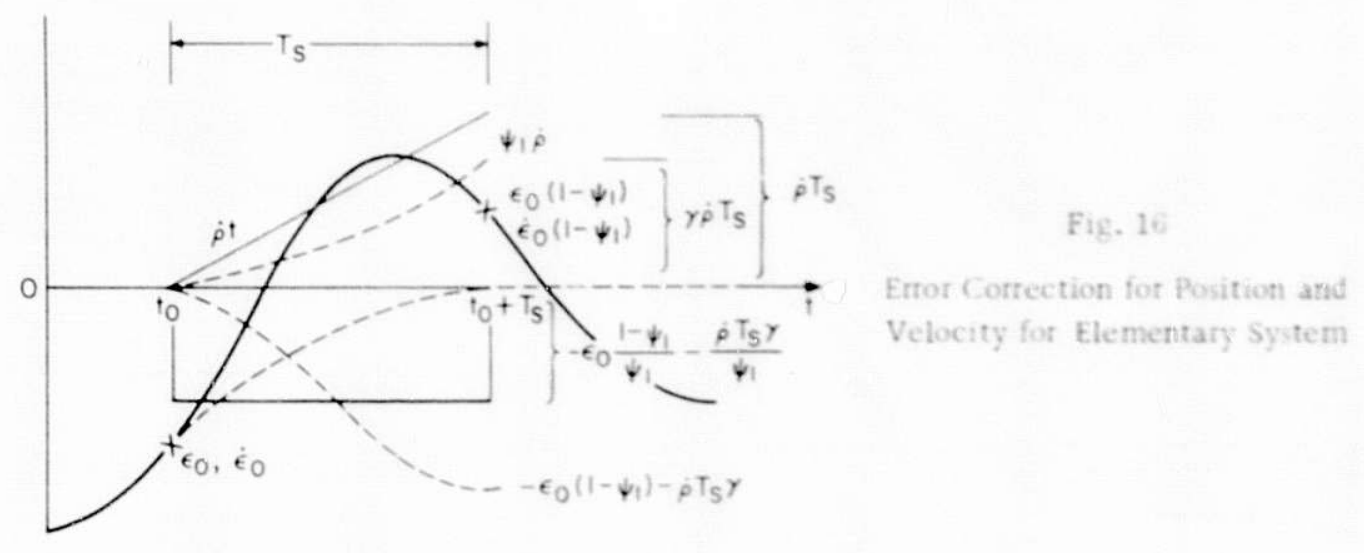

Application of the correction ramp causes an additional positional error at $t_{0}+T_{S}$ of value $\dot{p} T_{S} \%$. Therefore the total step-correction input must correct both for this error and for the future error $\varepsilon_{0}(1-t)$. That is,

$$
\psi_{1} \rho=-\dot{\rho} T_{S}{ }^{\nu}-\varepsilon_{0}\left(1-\psi_{1}\right),
$$

or

$$
\rho=\dot{\varepsilon} \frac{1-\psi_{1}}{\psi_{1}} \frac{\gamma T_{S}}{\psi_{1}}-\epsilon_{0} \frac{1-\psi_{1}}{\psi_{1}} .
$$

At $t=t_{0}+T_{S}$, the error magnitude and rate are both zero, and the stored energy in the system is zero. Thus, if the input correction signals are reduced to zero at $t=t_{0}+T_{S}$, the system output error will remain at zero for $t \geq t_{0}+T_{S}$.

When this scheme is applied to an underdamped system, the error history might appear as shown in Fig. 17. Assume a high-voltage power supply in which a step change in voltage is demanded at $t_{1}$. Then the step-correction signal will cause a smooth reduction of the error to zero in a time interval $\mathrm{T}_{\mathrm{s}}$. At $t=t_{2}$, a high-voltage spark causes an error signal to begin which goes unnoticed until $t_{3}$. At $t_{3}$, a correction signal is applied that causes a smooth reduction of the error and its rate to zero in a time interval $T_{S}$.

Fig. 17

Possible Error Correction for High-voltage Power Supply

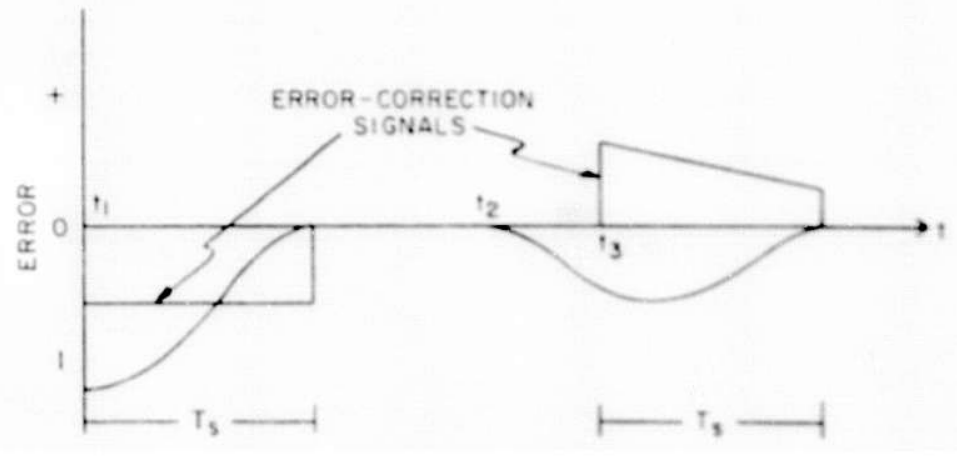


A possible mechanization is shown in Fig. 18. The switch across the ramp capacitor is to dump its charge at $T_{S}$ and thus to return the ramp to zero.

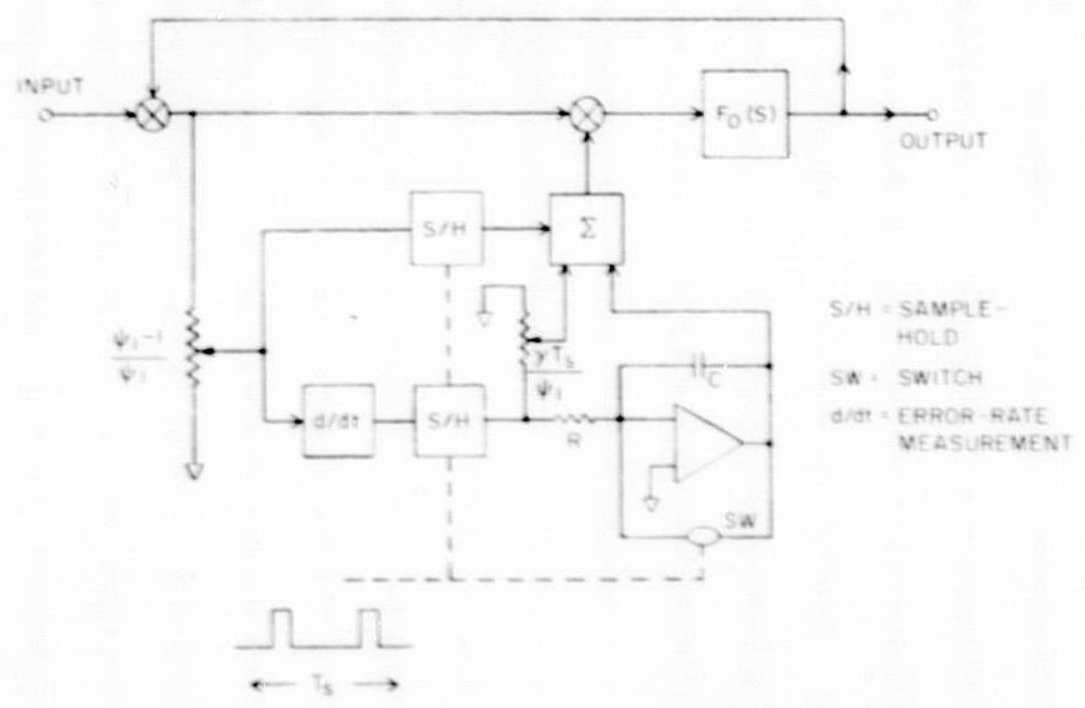

Fig. 18. Possible Mechanization for Positional and Velocity Error Correction

\section{Methods for Obtaining System Constants}

The three methods most useful are the step response method and two sinusoidal-response methods.

\section{Step-response Method}

A unit step is applied, and two quantities are measured as exactly as possible. These are:

a. The allowed settling time, $T_{\mathbf{S}}$. This is the time between application of the step and the first positive peak.

b. The dynamic gain, $\hat{y}_{1}$. This is the ratio of output to input at $T_{s}$, i.e., the value at the first positive peak.

The system constants can then be calculated as follows:

$$
\begin{aligned}
2 \alpha & =\frac{1}{T_{s}} 2 \ln \left(t_{1}-1\right), \\
\gamma & =\frac{2 T_{s} \ln \left(t_{1}-1\right)}{\left[\ln \left(t_{1}-1\right)\right]^{2}+\pi^{2}}, \\
\gamma & =1-t_{1} \frac{T}{T_{s}},
\end{aligned}
$$

etc. (See Fig. 19.) 


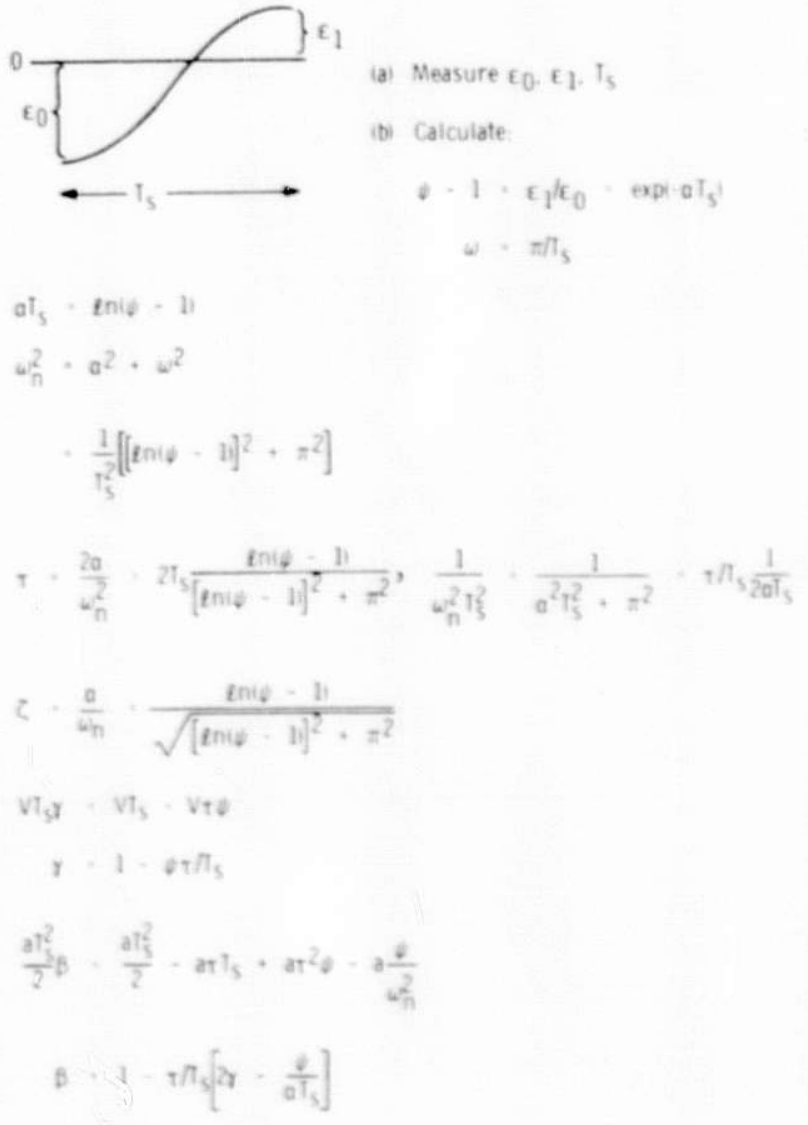

Overall Check

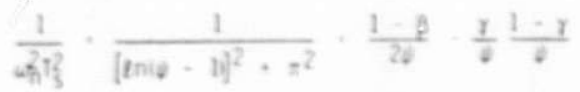

Fig. 19. Derivation of Formulas $6 \pi$ Calculating Values Shown in Table 11 and Fig. 20
Alternatively, the values can be obtained from a chart as shown in Table II or from curves, as shown in Fig. 20.

\section{Sinusoidal-response Methods}

a. Method 1: Measurement Made at $\omega_{n}$. The natural resonance frequency, $x_{n}$, is determined by observing the phase difference between the output and input signals. For the $F_{0}(\mathrm{~s})$ system, it is $90^{\circ}$ at ${ }^{\prime} n$, as shown in Fig. 21. With $\omega_{n}$ thus established, the gain, $E_{0} / E_{i n}$, is measured. The formulas shown in Fig. 21 then allow determination of the system constants. This method is particularly useful for systems that have a broad peak response. If a chart or curves similar to those in Fig. 20 are available, then only 5 must be determined. Locating its value on the chart or curves provides the other values.

b. Method 2: Measurement at Peak Response. The frequency ratio $\omega / \omega_{n}$ where peak response occurs can be determined by setting $d\left(E_{0} / E_{i n}\right) /$ $d\left(x / x_{n}\right)=0$, as shown in Fig. 22. This shows that peak response occurs for $w / x_{n}=\sqrt{1-2 S^{2}}$. Measurement of the peak response $\left(E_{0} / E_{\text {in }}\right)_{\max }=G_{\max }$ thus allows calculation of a value for $\zeta$ and (using charts or curves) determination of values for the other system constants.

\section{Summary of Describing Functions for $F_{0}(s)$ System}

Table II summarizes describing functions for the $\mathrm{F}_{0}(\mathrm{~s})$ system. Values for $2 \alpha T_{s}, \tau / T_{s}, \mathcal{C}, \gamma, \beta,\left(E_{o} / E_{i n}\right) \omega_{n},\left(E_{o} / E_{i n}\right)_{\max }, \omega_{n} / \omega_{n}$, and $\omega_{n} T_{s} \operatorname{can}$ be determined from any of three measurements:
a. Measurement of $\psi_{1}$ and $T_{S}$.
b. Measurement of $\left(E_{0} / E_{i n}\right) \omega_{n}$ and $\omega_{n}$.
c. Measurement of $\left(E_{0} / E_{i n}\right)_{\max }$ and $\omega$. 
TABLE 11. Describing Parameters for the $F_{0}(s)$ System

\begin{tabular}{|c|c|c|c|c|c|c|c|c|c|}
\hline 3 & $20 \mathrm{Ts}_{\mathrm{s}}$ & $+/ T_{s}$ & $c$ & y & A & $\left(E_{o} / E_{i n}\right) u_{n}$ & $\left(E_{0} / E_{\text {in }}\right)_{\max }$ & $w / w_{n}$ & $w_{n} T_{s}$ \\
\hline 1.01 & 9.210 & 0.296374 & 0.82608 & 0.70066 & 0.51968 & 0.5021 & 0.900 & 0.5636 & 5.574 \\
\hline 1.05 & 5.9915 & 0.31795 & 0.69011 & 0.66615 & 0.46495 & 0.7245 & 1.001 & 0.7237 & 4. 341 \\
\hline 1.1 & 4.6052 & 0.30354 & 0.59116 & 0.66610 & 0.45061 & 0.8458 & 1.049 & 0.8066 & 3.895 \\
\hline 1.2 & 3.21888 & 0.25834 & 0.45595 & 0.68999 & 0.45088 & 1.097 & 1.232 & 0.8900 & 3.530 \\
\hline 1.3 & 2.4079 & 0.21273 & 0.35785 & 0.72345 & 0.46250 & 1.400 & 1. 500 & 0.9340 & 3.364 \\
\hline 1.4 & 1.83258 & 0.17112 & 0.27999 & 0.76043 & 0.47829 & 1.786 & 1.860 & 0.9600 & 3.273 \\
\hline 1.5 & 1.3863 & 0.13394 & 0.21515 & 0.7991 & 0,49608 & 2.321 & 2.377 & 0.9765 & 3.217 \\
\hline 1.6 & 1.02165 & 0.10085 & 0.1605 & 0.8386 & 0.515 & 3.115 & 3.156 & 0.9870 & 3.183 \\
\hline 1.7 & 0.713350 & 0.071358 & 0.11281 & 0.87869 & 0.5345 & 4.432 & 4.461 & 0.9936 & 3.162 \\
\hline 1.8 & 0.446287 & 0.045 & 0.07085 & 0.9190 & 0.5544 & 7.057 & 7.075 & 0.9975 & 3.149 \\
\hline 1.9 & 0.210721 & 0.02133 & 0.03352 & 0.95948 & 0.5745 & 14.92 & 14.92 & 0.9994 & 3.143 \\
\hline 1.95 & 0.1025866 & 0.01039 & 0.016325 & 0.97974 & 0.5846 & 30.63 & 30.62 & 0.9999 & 3.142 \\
\hline 2.00 & 0 & 0 & 0 & 1.0 & 0.5947 & $\infty$ & $\infty$ & 1.0 & $\pi$ \\
\hline
\end{tabular}

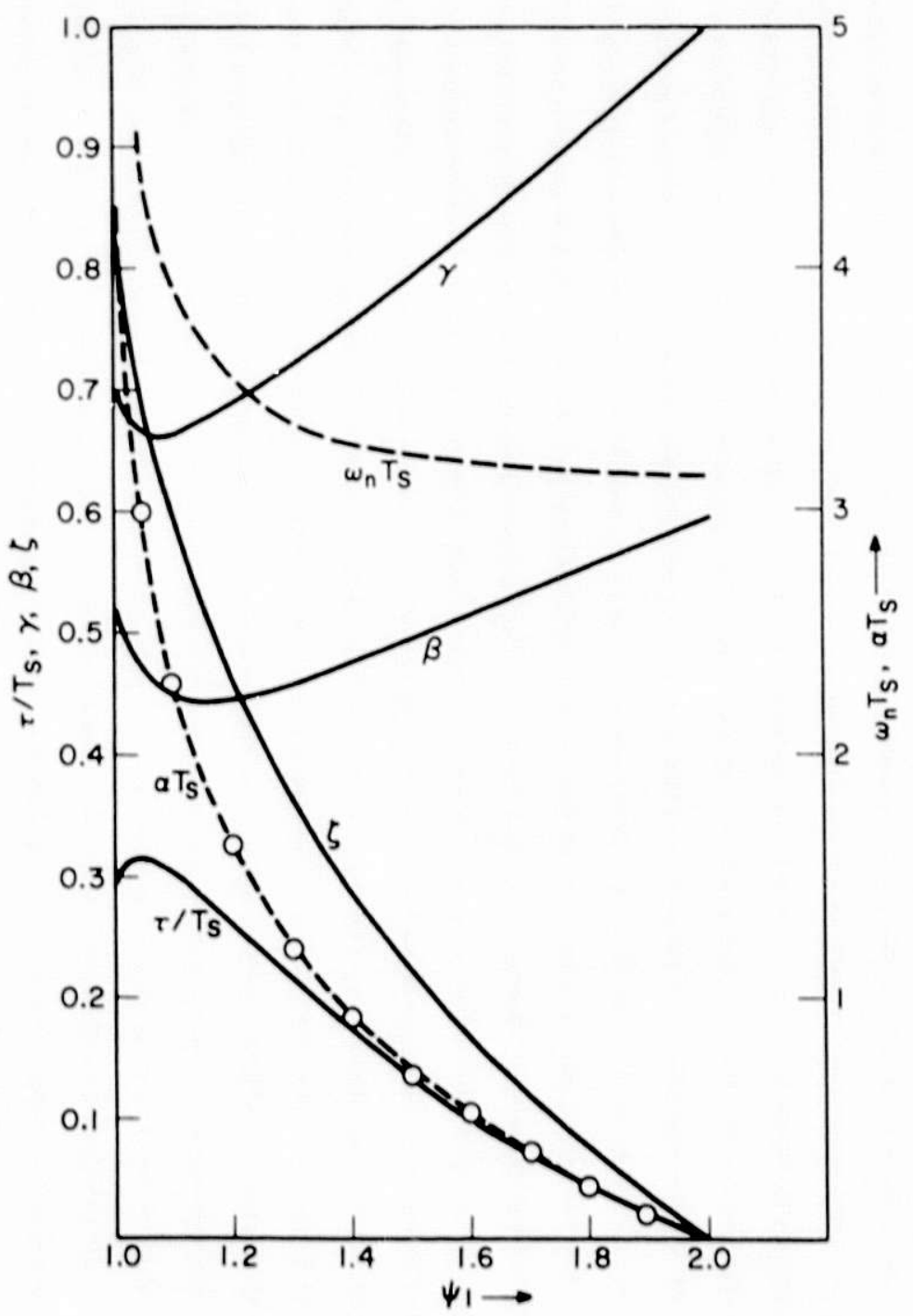

Fig. 20. Parameters for $F_{0}$ (s) System when $\psi_{1}$ and $T_{5}$ Are Known 


$$
\begin{aligned}
& \frac{E_{0}}{E_{\text {in }}}(\omega) \cdot \frac{1}{\sqrt{\left[1-\omega^{2} / \omega_{n}^{2}\right]^{2}+4 \zeta^{2} \omega^{2} / \omega_{n}^{2}}} \\
& \theta \cdot \tan ^{-1} \frac{\omega \tau}{1 \cdot \omega^{2} / \omega_{n}^{2}} \\
& \text { at } \omega=\omega_{n} \\
& \frac{i_{0}}{E_{\text {in }}} \cdot \frac{1}{2 \zeta} \cdot \frac{1}{\omega_{n} T} \cdot \frac{\omega_{n}}{2 a}, \theta=-90^{\circ} \\
& \therefore z=\frac{1}{2 E_{0} t_{\text {in }}} \\
& \mathrm{I}_{5} \cdot \pi / \omega \cdot \frac{\pi}{\omega_{n} \sqrt{1-\zeta^{2}}} \\
& \text { 2a } \cdot \frac{\omega_{n}}{E_{d} t_{i n}} \cdot \frac{\pi}{T_{s} \sqrt{1-\zeta^{2} E_{0} d t_{i n}}} \\
& T \cdot \frac{1}{\omega_{n} E d t_{\text {in }}} \\
& \text { a } T_{5} \cdot \frac{\sqrt{2}}{2 \sqrt{1-\zeta^{2}} E_{d} t_{\text {in }}} \\
& \psi_{1}+1+\text { expi-a } \mathrm{I}_{\mathrm{s}} \text { ) } \\
& \gamma \cdot 1-4 \pi / \pi_{S} \\
& \beta \cdot 1+\tau_{1 / 5}\left[2 \gamma+\frac{\phi_{1}}{a T_{s}}\right]
\end{aligned}
$$

Fig. 21

Determination of System Constants from Sinusoidal Response at $\omega=\omega_{n}$

For a sinusoidal input, the response of the $\mathrm{F}_{0}(\mathrm{~s})$ system is

$$
\frac{E_{0}}{E_{\text {in }}}=\frac{1}{\sqrt{1-2 \frac{u^{2}}{u_{n}^{2}}+\frac{u^{4}}{u_{n}^{4}} \cdot 4 z^{2} \frac{u^{2}}{u_{n}^{2}}}}
$$

The value of u/un yielding maximum response is found by differentiating Eq. 22.1 and setting the result equal to zero, This yields

$$
\frac{u(\max )}{u_{n}} \cdot \sqrt{1-2 Z^{2}}
$$

Using Eq. 22.2 in Eq. 22.1 and squaring yieids

$$
\left(\frac{E_{0}}{E_{\text {in }}}\right)_{\max }^{2} \cdot G_{\max }^{2} \cdot \frac{1}{4 \zeta^{2} 11 \cdot \zeta^{2}}
$$

Fig. 22

Determination of System Constants from Peak Sinusoidal Response

and

$$
c \cdot \frac{1 \pm \sqrt{1-\frac{1}{G_{\max }^{2}}}}{2}
$$

For $6 \rightarrow \infty, \zeta \rightarrow 0$; thus.

$$
\begin{aligned}
& c \cdot \frac{1 \cdot \sqrt{1 \cdot \frac{1}{6_{\text {max }}^{2}}}}{2} \\
& u_{n} \cdot \frac{u_{\max }}{\sqrt{1 \cdot 2 \zeta^{2}}} \\
& a \cdot u_{n} \\
& T_{s} \cdot \frac{\pi}{u} \cdot \frac{\pi}{u_{n} \sqrt{1 \cdot \zeta^{2}}}
\end{aligned}
$$

etc. 


\section{GENERAL APPLICATION}

A. System Elements and Modeling

Some of the most generally used system elements are shown in Fig. 23 with their symbols. The triangular objects are integrated circuit operational amplifiers.

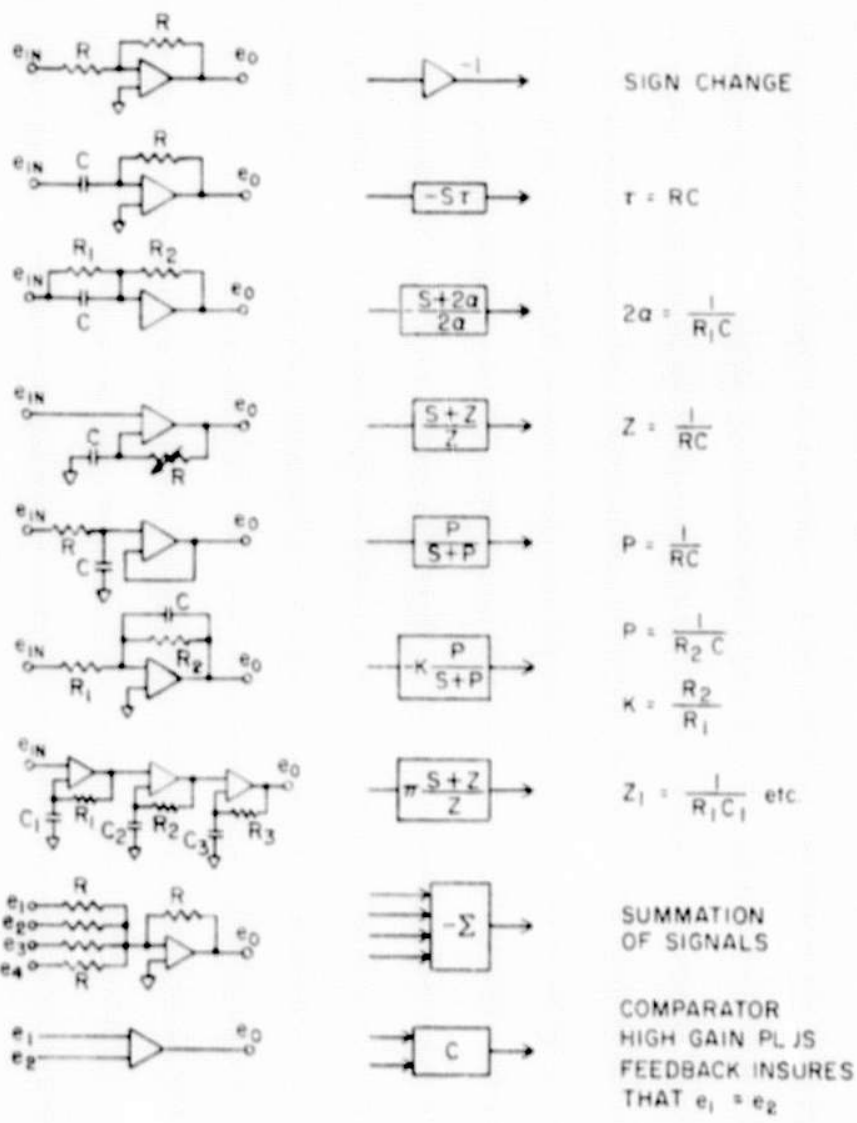

Fig. 23

Some Basic System Elements

Figure 24 shows examples of the many ways the basic system elements can be connected to provide specified transfer functions.

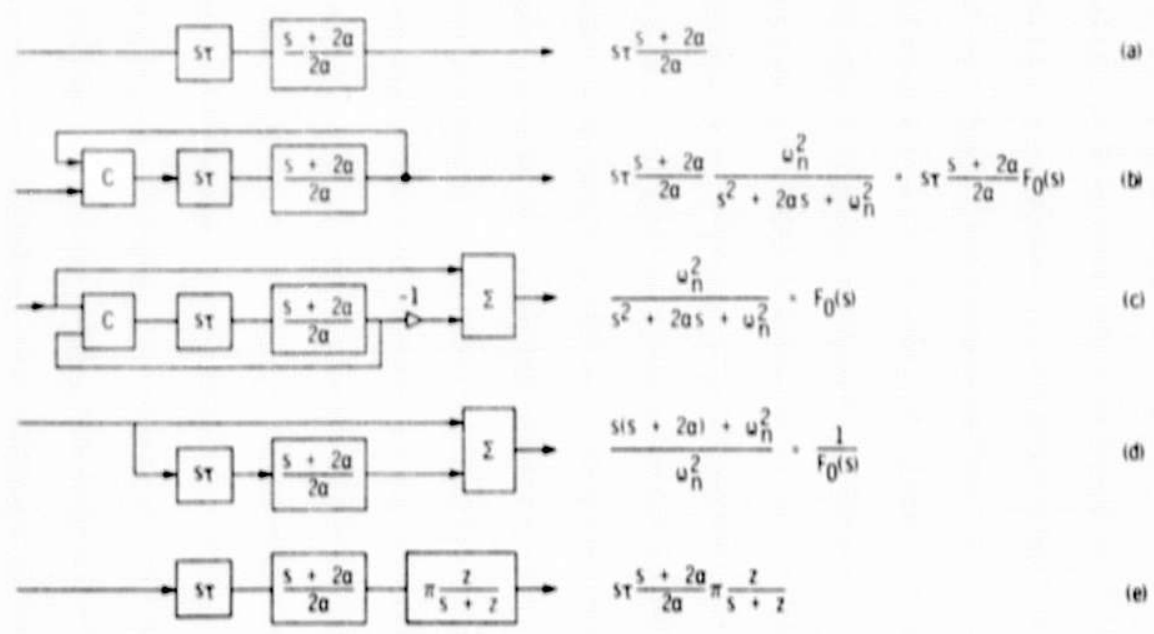

Fig. 24. System Modeling with Basic Elements 
B. First-order Systems

A first-order system with a transfer function $\mathrm{p} /(\mathrm{s}+\mathrm{p})$ will not overshoot or ring, and one must dictate an allowed settling time, $\mathrm{T}_{\mathbf{S}}$. Figure 25 shows the derivation for the required step-input correction, which is seen to correspond closely with that for the elementary second-order system. The correction device is shown in Fig. 25 b.

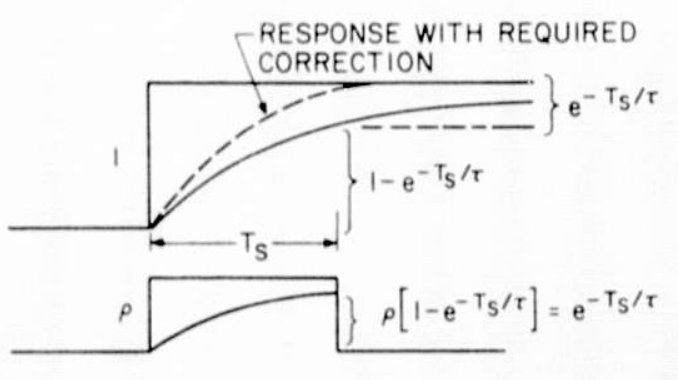

$$
\rho=\frac{e^{-T_{S} / \tau}}{1-e^{-T_{S} / \tau}}=\frac{1-\psi_{1}}{\psi_{1}}
$$

(a) STEP CORRECTION

Fig. 25

Error Correction for the $p /(s+p)$

First-order System

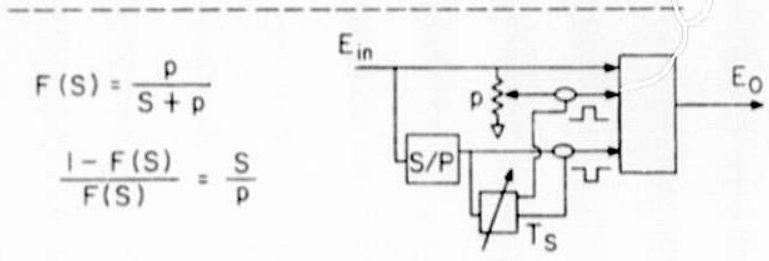

(b) OVERALL CORRECTION

In some cases, it may be advantageous to convert a second-order sys tem to a first-order system and to apply adaptive controls based on prediction techniques, using a time-shared digital computer. The technique is described in Sec. IX of this report.

C. More Complex Systems

1. $\underline{F}_{0}(\mathrm{~s})$ Systems with Zeros

a. Velocity-corrected Positional Servo Systems. The most commonly encountered $F_{0}(s)$ system with a zero is the so-called velocity-corrected positional servo. It has the transfer function

$$
F(s)=\frac{s+z}{z} F_{0}(s)
$$

For proper velocity correction, the value of $z$ is chosen to be equal to $1 / \tau$. By algebraic expansion and with $z=1 / \tau$, Eq. 33 becomes

$$
F(s)=F_{0}(s)+s \tau F_{0}(s) \text {. }
$$


The response to an input ramp, $\mathrm{V} / \mathrm{s}^{2}$, is

$$
E_{O}(s)=\frac{\mathrm{VF}_{0}(s)}{s^{2}}+T \frac{\mathrm{VF}_{0}(\mathrm{~s})}{\mathrm{s}}
$$

That is, it is the sum of an $F_{0}(s)$ system response to a ramp V plus the response of the $F_{0}(s)$ system to a step $V \tau$. Using the equations shown in Fig. 4, we can write, for $E_{O}(t)$,

$$
\begin{aligned}
E_{0}(t)= & V t-V \tau\left[1-e^{-\alpha t}(\cos \omega t+\delta \sin \omega t)\right]-\frac{V}{\omega_{n}^{2}} \frac{\omega_{n}^{2}}{\omega} e^{-\alpha t} \sin \omega t \\
& +V \tau\left[1-e^{-\alpha t}(\cos \omega t+\delta \sin \omega t)\right]=V t-\frac{V}{\omega_{n}^{2}} \frac{\omega_{n}^{2}}{\omega} e^{-\alpha t} \sin \omega t .
\end{aligned}
$$

Equation 36 shows that this system exhibits no steady-state error for a ramp input. The response to an input parabola, $a / \mathrm{s}^{3}$, is

$$
E_{0}(s)=a \frac{F_{0}(s)}{s^{3}}+a T \frac{F_{0}(s)}{s^{2}}
$$

and

$$
E_{0}(t)=\frac{a t^{2}}{2}-\frac{a}{\omega_{n}^{2}}\left[1-e^{-\alpha t}(\cos \omega t+\delta \sin \omega t)\right]
$$

The response to an input step, $\mathrm{P} / \mathrm{s}$, is

$$
E_{0}(t)=P\left[1-e^{-\alpha t}(\cos \omega t+\delta \sin \omega t)\right]+\tau P \frac{\omega_{n}^{2}}{\omega} e^{-\alpha t} \sin \omega t
$$

The system is more lively than the $F_{0}(s)$ system because of the second term in Eq. 39. The time between application of an input step and the first positive peak is a shorter period than the time, $T_{S}$, between succeeding maxima and minima. If this time is designated $T_{S}^{\prime}$ and the peak $\psi_{1}^{\prime}$, the system response to a step can be compared with that for an $F_{0}(s)$ system and results in Table III. It is seen that the shortening of the initial time $\mathrm{T}_{\mathrm{S}}^{\prime}$ is the rnost sensitive indication of the presence of the zero. The interval between successive maxima and minima is the time to be used for $T_{\mathbf{S}}$.

When the output due to a step input of $\mathrm{P}$ is measured at a time $\mathrm{T}_{\mathbf{S}}$ after application of the input, the output value is $\mathrm{P} \psi_{1}$ (see Eq. 39). Using this value for $\psi_{1}$ as shown in Fig. 26 yields a response that is correct for $t \geq T_{S}$ as is detailed in Fig. 27. The value for $\gamma$, measured at $T_{S}$ will be zero as is evident from Eq. 36. The value for $\beta$ is not zero as can be seen from Eq. 38 . 
TABLE III. Characteristics of the Velocity-corrected

Positional System

\begin{tabular}{cccc}
\hline 5 & $\psi_{1}$ & $\psi_{1}^{\prime}$ & $T_{S}^{\prime} / T_{S}$ \\
\hline 0.707 & 1.043 & 1.21 & 0.5 \\
0.456 & 1.20 & 1.28 & 0.8 \\
0.28 & 1.40 & 1.47 & 0.82 \\
0.16 & 1.60 & 1.63 & 0.9
\end{tabular}

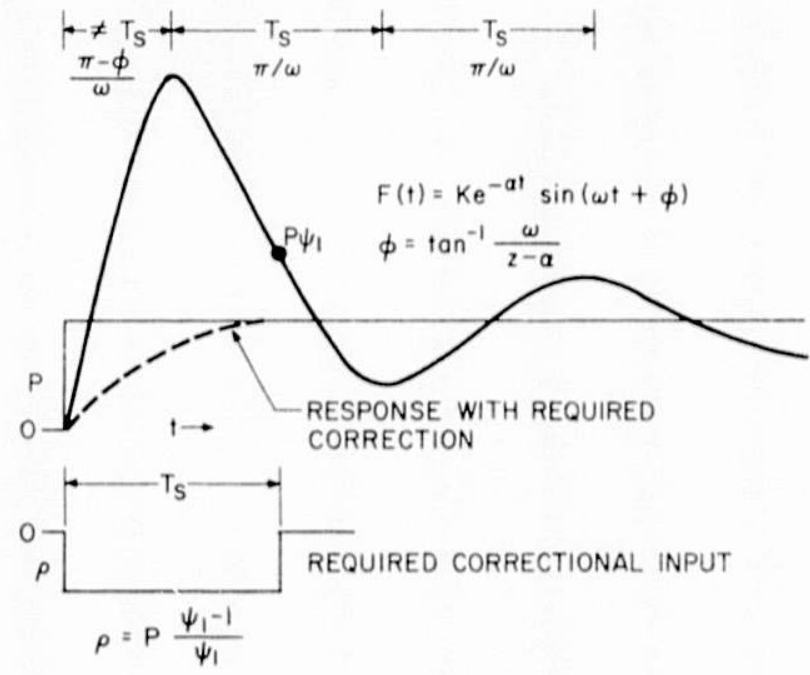

Fig. 26. Step Response of the $\mathrm{F}_{0}(\mathrm{~s})$ System with Zeros

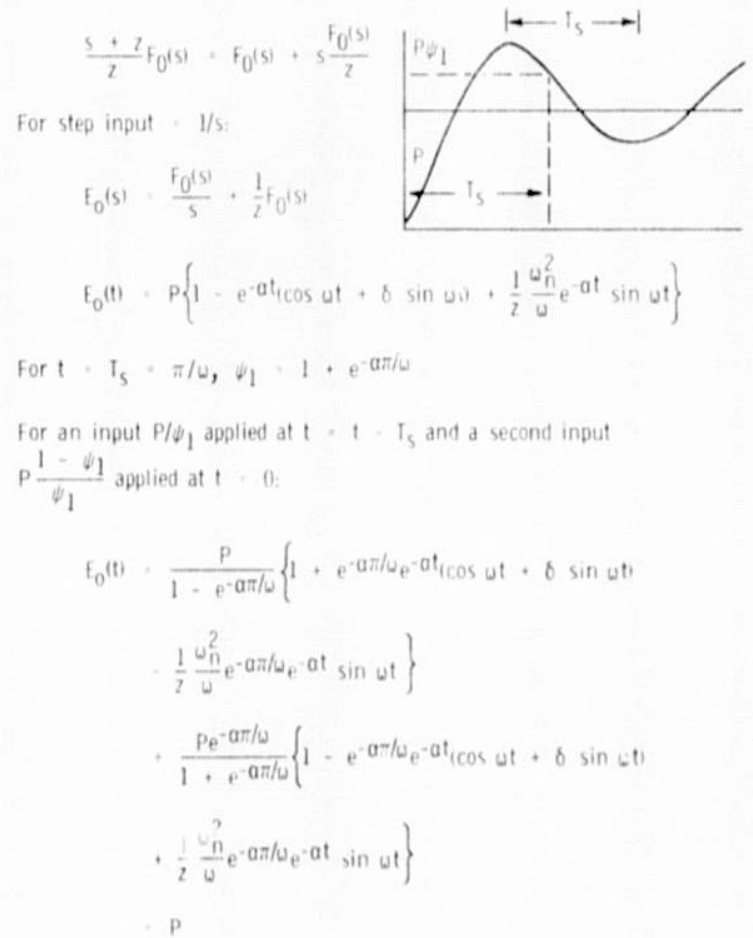

By similar reasoning, correct ramp and parabolic response is shown to be obtained by using the techniques for the $\mathrm{F}_{0}(\mathrm{si})$ system it $\mathrm{T}_{\mathrm{s}}$ is chosen as shown above.

Fig. 27. Step Response of Velocity-corrected Positional System When Using Control Techniques

It is worth noting here that the so-called velocity-corrected positional servo is velocity-corrected only in the steady state, i.e., after the transient term of Eq. 36 decays to zero. Comparison with the correction shown in Fig. 7 shows that, through use of the subject theory, the system of Fig. 3 is velocity-corrected for all times. It is also worth noting that the improvement in velocity response has degraded the positional response, although it has improved the acceleration response. Correctional signals can be generated to 
provide good positional response but only at $t \geq T_{s}$, i.e., the same as for a system not velocity-corrected. This fact hints at a theorem whose desirability will become evident in later sections. It is stated here for reference: One approach to the control of a system describable as $[\pi(s+z) / z] F_{0}(s)$, or $[\pi \mathrm{p} /(\mathrm{s}+\mathrm{p})] \mathrm{F}_{0}(\mathrm{~s})$ is to insert appropriate time constants at the input so as to convertitto an $\mathrm{F}_{0}(\mathrm{~s})$ system, and to then apply the methods described in Sec. V of this report to achieve optimum control. A correction device for this function is shown in Fig. 30 (later).

b. $F_{0}(s)$ System with Arbitrary Zeros. These systems can be described as $F(s)=[\pi(s+z) / z] F_{0}(s)$. Analysis of the response of such systems reveals that the percentage overshoot can be very large, perhaps several hundred percent. If the input is well defined, the appropriate correction can be determined. For example, if the system under consideration will receive only step inputs, the program of Fig. 26 is sufficient. If precise response is required for arbitrary inputs then the corrector of Fig. 28 must be used. The $z /(s+z)$ circuits convert to the $F_{0}(s)$ system, which can then be controlled by application of the theory presented in Sec. V above.

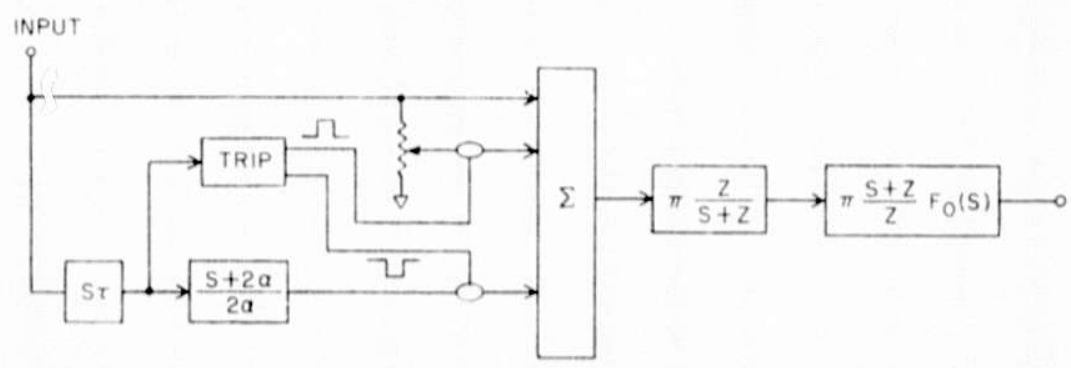

Fig. 28. Overall Correction for $F(s)=\pi[(s+z) / z] F_{0}(s)$ System

\section{2. $\mathrm{F}_{0}(\mathrm{~s})$ System with Real Poles}

This system is less lively than the $F_{0}(\mathrm{~s})$ system. This is due to the first-order exponential terms (the second term of Eq. 3) and the reduction of amplitude of the oscillatory term (see Appendix). (See, for example, Fig. 36 later.) The system response to a step input does not exhibit dynamic symmetry in amplitude, and it is impossible to derive a simple value for $p$ as shown in Fig. 8 for step-input correction. (A sequential step program will be shown later in Fig. 36.) It can be seen that lack of symmetry in amplitudes is much more pronounced than lack of symmetry in time. The simplest method for applying error correction, as well as for increasing the speed of response of the system, is to convert it to an $F_{0}(s)$ system. This is illustrated in Fig. 29, where the added $\pi(s+p) / p$ circuit response cancels the system $\pi p /$ ? $+p)$ re sponse. The input signal is applied through the $F_{0}(s)$ signal-correction system previously described. 


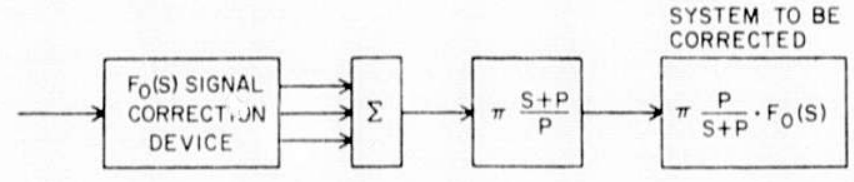

Fig. 29. Method for Converting $\pi[\mathrm{p} /(\mathrm{s}+\mathrm{p})] \mathrm{F}_{0}(\mathrm{~s})$ System Response to That of the $\mathrm{F}_{0}(\mathrm{~s})$ Response

\section{Correction of Complex Systems}

The error-correction unit for a complex system must contain the $\pi z /(s+z)$ elements of Fig. 28, the $\pi(s+p) / p$ elements of Fig. 29 and the $\mathrm{s}(\mathrm{s}+2 \alpha) / 2 \alpha$ elements of Fig. 5, as well as elements to control complex zeros and minor complex poles.

steps:

Overall correction of a complex system then proceeds in three

a. The response to a step input is observed. The maxima and minima, $\psi_{1}, \psi_{2} \ldots$, are recorded, as well as their times of occurrence, $\mathrm{T}_{\mathbf{S}}(1)$, $\mathrm{T}_{\mathrm{S}}(2) \ldots$. Lack of symmetry reveals the presence of poles and/or zeros other than those inherent in an $F_{0}(s)$ system. The most sensitive indicator of the presence of a dominant zero is the time interval $\mathrm{T}_{\mathbf{S}}(1)$ as compared with $\mathrm{T}_{\mathbf{S}}(2)$, $\mathrm{T}_{\mathrm{S}}(3) \ldots$. The most sensitive indicator of a dominant real pole is the absence of symmetry for the relation $\left(1-\psi_{1}\right)^{n}=1-\psi_{n}$.

b. When the step-response data have been thoroughly analyzed, the error-correction unit is inserted at the system input. A series of step inputs is made and the appropriate "depoling" and "dezeroing" controls are manipulated until dynamic symmetry exists. When this is accomplished, the complex system has been converted to an $\mathrm{F}_{0}(\mathrm{~s})$ system.

c. Measurements of $\mathrm{T}_{\mathrm{S}}, \psi, \beta$, and $\gamma$ are then made and appropriate corrections made, according to Sec. V of this report.

\section{ERROR-CORRECTION DEVICES}

A. Real-pole and Real-zero Correction

A circuit for pole or zero cancelation is shown in Fig. 30. In Fig. 30a, the switch is actuated so the transfer function is $(s+z) / z$ and thus cancels a pole. In Fig. 30b, the switch is actuated so the transfer function is $\mathrm{p} /(\mathrm{s}+\mathrm{p})$ and cancels a zero.

\section{B. Correction Unit for $F_{0}(s)$ and $1 / F_{0}(s)$ Systems}

Figure 31 shows a unit that can be used for error correction of $F_{0}(s)$ systems or for $1 / F_{0}(s)$ systems. Figure 32 is a circuit diagram of such a unit without step correction, and Fig. 33 is a circuit diagram of a unit with step correction. 

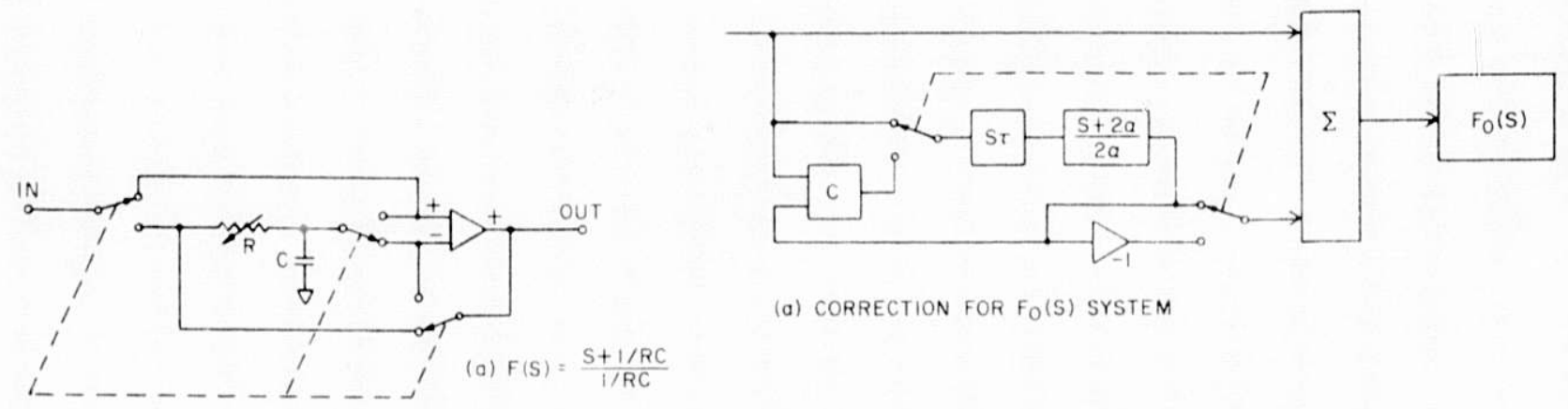

(a) CORRECTION FOR FO(S) SYSTEM

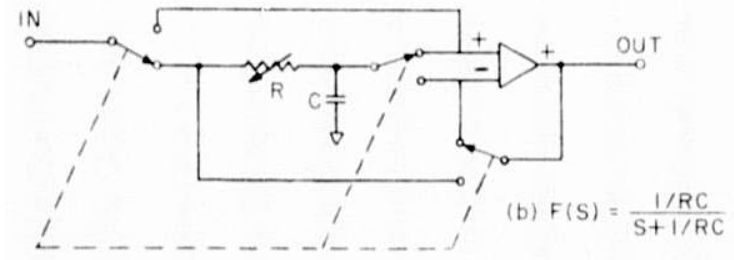

Fig. 30. Pole- and Zero-correcting Unit

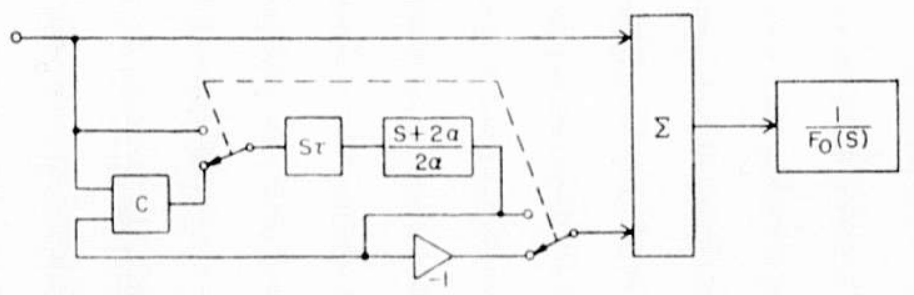

(b) CORRECTION FOR $\frac{1}{F_{0}(S)}$ SYSTEM

Fig. 31. Unit for Correcting Either $\mathrm{F}_{0}(\mathrm{~s})$ or $1 / \mathrm{F}_{0}(\mathrm{~s})$ System

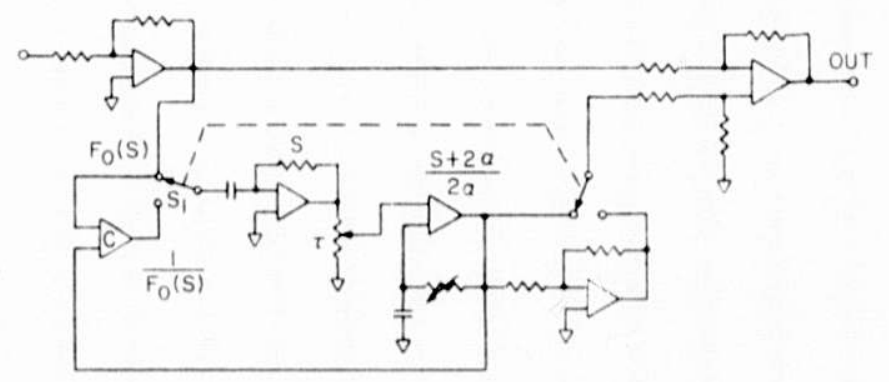

Fig. 32

Unit for Correcting Either $\mathrm{F}_{0}(\mathrm{~s})$ or $1 / \mathrm{F}_{0}$ (s) System without Step Correction

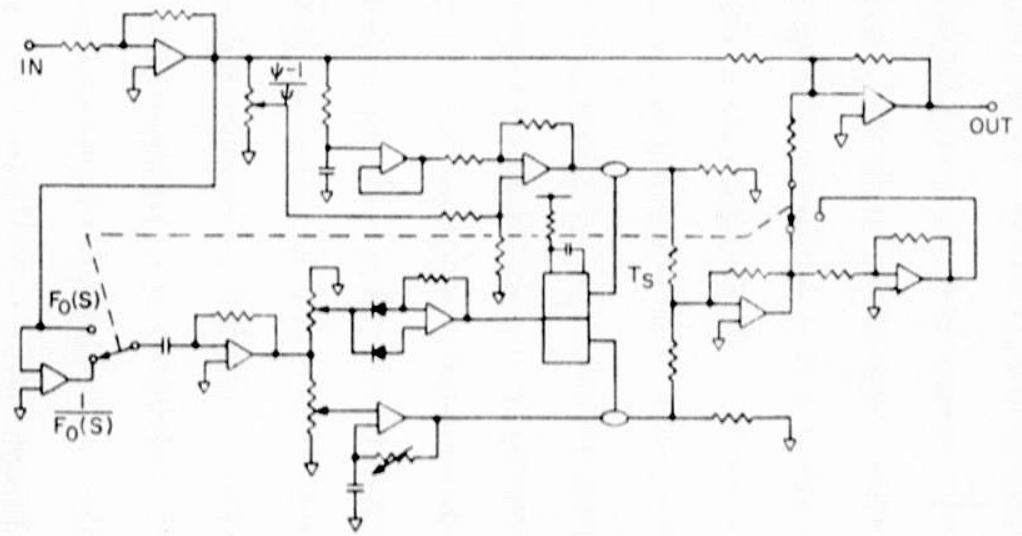

Fig. 33. Unit for Correcting Either $\mathrm{F}_{0}(\mathrm{~s})$ or $1 / \mathrm{F}_{0}(\mathrm{~s})$ System with Step Correction 
C. Portable Units for Determination of Error-correction Requirements for

Figure 34 shows the block diagram for a portable unit to be used for determining correction-unit requirements for open-loop systems. It consists of one unit of Fig. 33, one unit of Fig. 32, and three units of Fig. 30. Thus, it may be used with systems as complex as, for example,

$$
F(s)=\frac{K\left(s+z_{1}\right)\left(s^{2}+2 \alpha_{1} s+w_{n_{1}}^{2}\right)}{\left(s+p_{1}\right)\left(s+p_{2}\right)\left(s^{2}+2 \alpha_{2} s+w_{n}^{2}\right)} .
$$

Figure 35 shuws the block diagram for a portable unit to be used in investigating closed-loop systems.

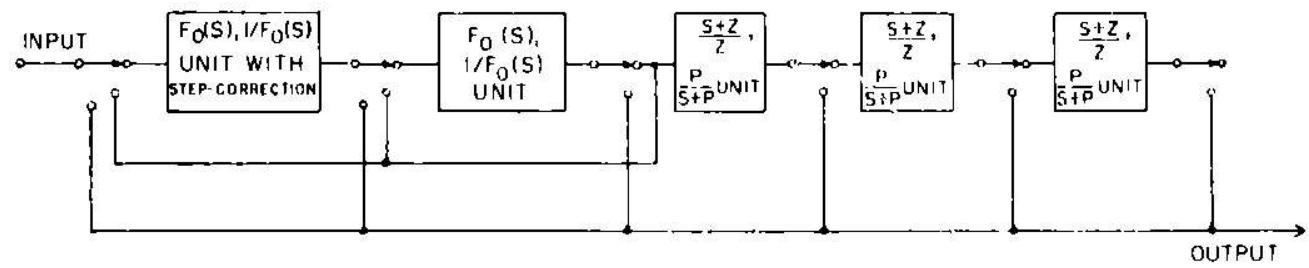

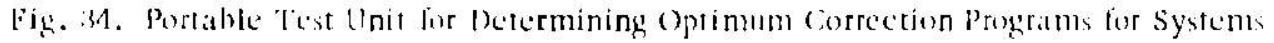

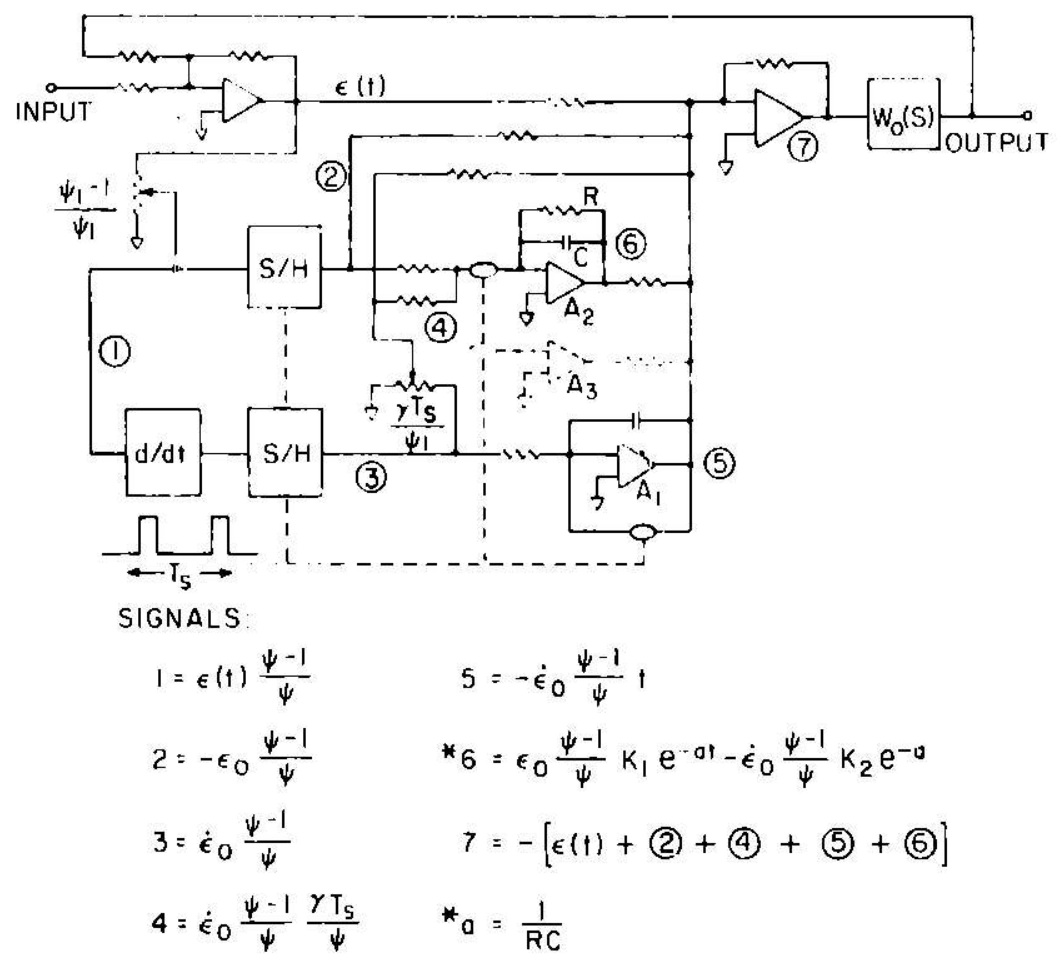

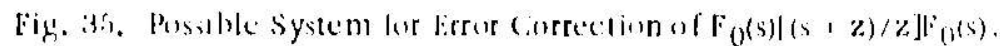
and $\mid p /(s \quad p) f_{0}(i)$ Systens. Adelitional amplificers, $A_{3} \ldots$ etc., maly be alded tu cormpensille for additional poles. 
VIII. DIGITAL CÓNTROL

\section{A. General Application}

The performance of tape-controlled machines can be improved by application of the prescnt control method. Many of the machines are stepprogrammed; that is, eithe $r$ a tool head or a carriage is translated from point to point in $\mathrm{x}$ and $\mathrm{y}$ for drilling and/or tapping of holes, riveting, spot-welding, punching, etc. If the system gain can be increased and response time improved as described in Sec. V.A.2 above, the digital program can be changed to achieve rapid precise control, thus improving production capacity. Ramp control can be substantially improved by adding to the programmed signal, Vt, a step of vaiue VT.

A digital computer can be used to perform the error-correction action described in Sec. V.B. Referring to Fig. 12, the digital computer would occupy the box called "error-correction signals." It would operate upon the error signal, smoothing and curve fitting so that accurate values for $\varepsilon$ and $\dot{\epsilon}$ are estir.iated, even in the presence of noise. It would then generate the appropriate step and ramp values required by the theory.

Where large numbers of systems are used, such as a large ship, a large aircraft, or a large production facility, time sharing of a computer for a number of systems is possible. This is especially attractive where transfer functions change with time as, for example, a large aircraft climbing into less dense air and burning off fuel. Proper programming and calculations will provide for adaptive system response exhibiting superior control characteristics.

Some examples of adaptive control are described in Sec. IX below. Adaptive control of complex systems is described in the following paragraph.

\section{B. Sequential Control Programs}

Given a complex time-varying system whose precise control is sufficiently important that a computer can be dedicated, a sequential control program with continuous calculations of constants may prove of value. Such systems tend to have dominant real poles as well as dominant complex poles. Thus, the response to a step input might appear as shown in the example in Fig. 36 .

This sytem is composed of an $F_{0}(\mathrm{~s})$ system, modified by two simple poles at $\sigma=-0.648$ and $\sigma=-1.352$ and a pair of repeated zeros at $\sigma=-1$. (The system is described in detail on page 265 of Ref. 4.) The exponential transient due to the pole at $\sigma=-0.648$ persists for a much longer time than the time to the first positive peak, $\mathrm{T}_{\mathbf{S}}$. The system is "lively" only because of the presence of the repeated zeros at $Y=-1$. Nonetheless, the eifect of the pole is to cause the error to still be $-4 \%$ at $t=7 \mathrm{~T}_{\mathrm{s}}$. 


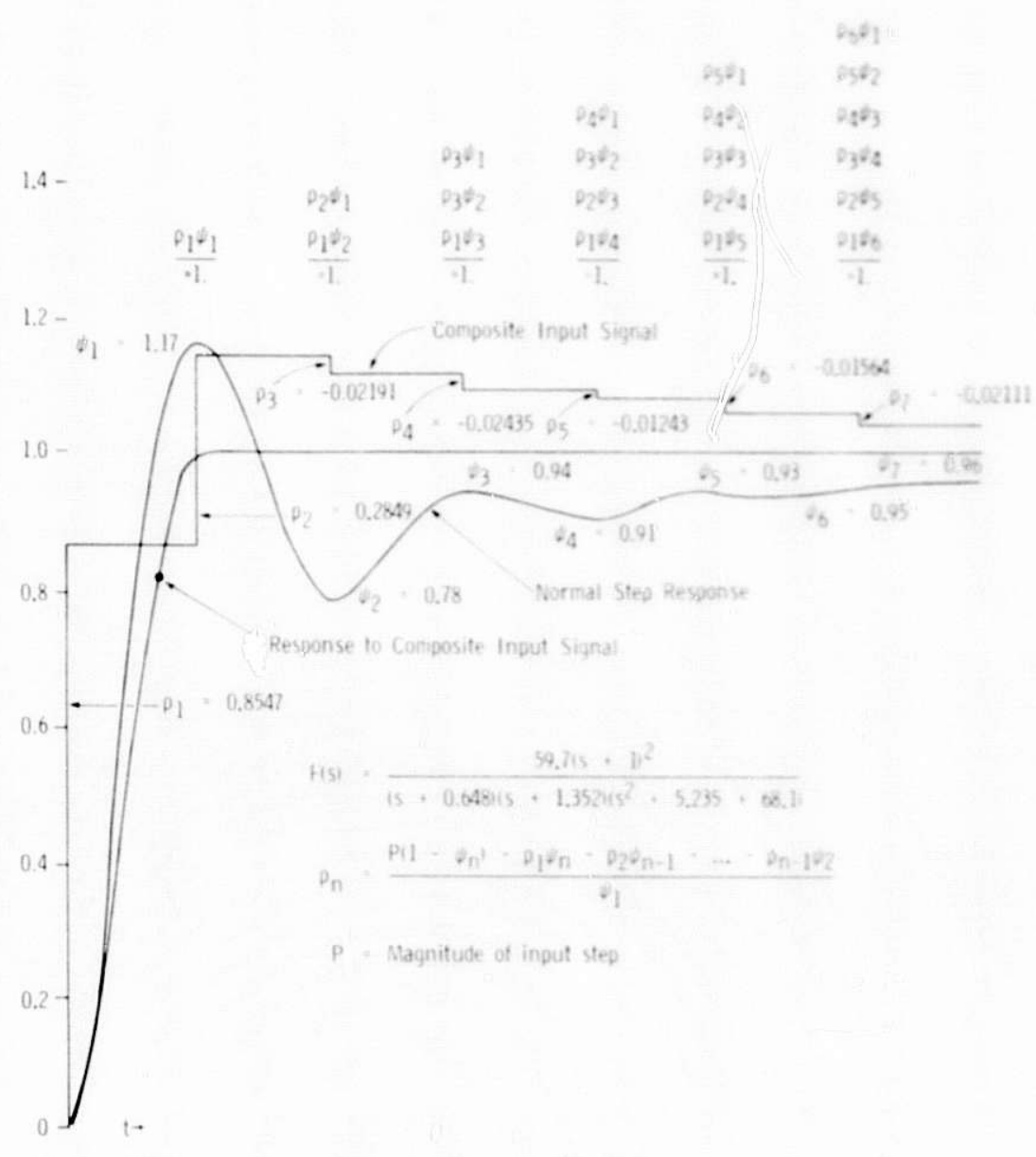

Fig. 36. Sequential Control Program tor Step Control of a Complex System

A single correctional input will not provide precise control. If the composite input signal shown is applied rather than the step, then good step control is achieved. The composite signal is the sum of a series of steps: $\rho_{1}$ applied at $t=0, \rho_{2}$ applied at $t=T_{S}, \rho_{3}$ applied at $t=2 T_{S}$, etc. The values $f_{n}$ can be determined as indicated at the top of Fig. 36. Values for $p_{n}$ may also be calculated from the general equation

$$
p_{n}=\frac{P\left(1-\psi_{n}\right)-p_{1} \psi_{n}-p_{2} \psi_{n-1}-\ldots-p_{n-1} \psi_{2}}{\psi_{1}} .
$$

Further analysis reveals that for improved ramp response to an input ramp Vt, a series of step inputs of magnitude V $L_{n}$ must be applied, where $L_{n}$ has the value

$$
L_{n}=\frac{V T_{N}\left(1-\gamma_{N}\right)-L_{1} \psi_{N}-L_{2} \psi_{N-1}-\ldots-L_{N-1} \psi_{2}}{\psi_{1}} .
$$

For a parabolic input, $\frac{1}{2} a t^{2}$, a series of ramps of value $a L_{n} t$ are applied as well as a series of steps, $a M_{n}$, where $M_{n}$ has the value 


$$
\begin{aligned}
M_{n}= & {\left[a \frac{T_{s}^{2}}{2}\left(1-\beta_{N}\right)-M_{N-1} \psi_{2}-M_{N-2} \psi_{3}-\ldots-M_{1} \psi_{N}-L_{1} T_{N} \gamma_{N}\right.} \\
& \left.-L_{2} T_{N-1} V_{N-1}-\ldots-L_{N} T_{1} \gamma_{2}\right] / \psi_{1} .
\end{aligned}
$$

Application of the sequential correctional program provides improved control of a complex system. For a time-varying system, response correctness will deteriorate with time. Measurement of response errors and appropriate computation allow modification to the correctional program and thus provide adaptive control.

\section{ADAPTIVE CONTROL THROUGH USE OF PREDICTION TECHNIQUES}

A. General

In many experimental situations, control-system characteristics are subject to arbitrary change. For example, Fig. 37 shows a liquid-temperature

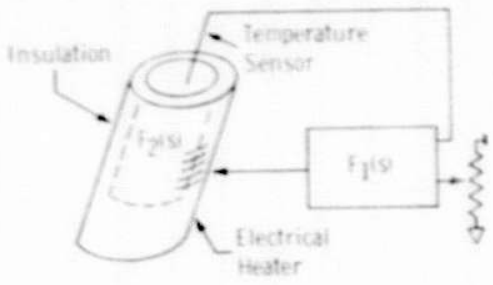

a) Lieuid-temperature Controllet

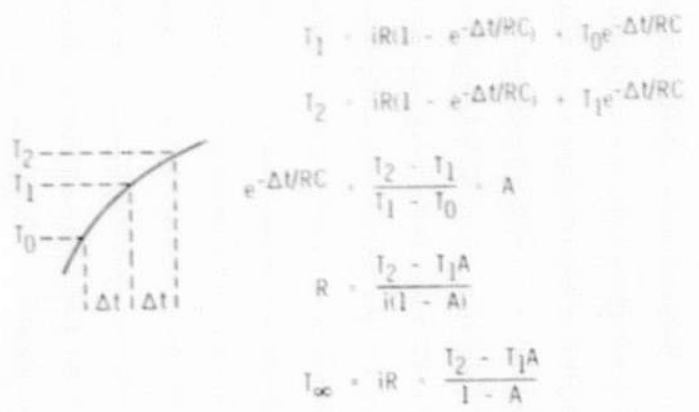

c) Calculation of Parameter $R$ and Prediction of Final Temperature.

To. by Time History

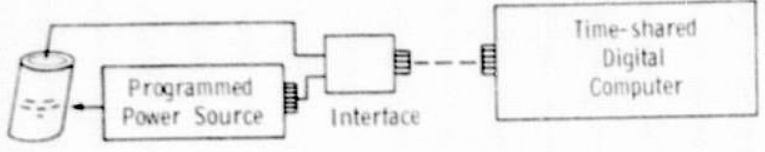

d) Control by Time-shared Digital Computer

Fig. 37. Analysis of Adaptive Control of Temperature of Liquid by Time-shared Digital Computer controller. The electrical analog of the heating pot is shown in Fig. 37b, where the capacitance C represents the heat capacity of the pot assembly plus the heat capacity of the contained liquid, $\mathrm{R}$ is the thermal leakage, $\mathrm{T}$ is the tem perature achieved, and $\mathrm{i}$ is the input electrical heater power. The transfer function is

$$
\begin{aligned}
F_{2}(s)= & \frac{T(s)}{i(s)}=i \frac{1 / C}{1 / R C+s} \\
& +C T_{0} \frac{1 / C}{1 / R C+s},
\end{aligned}
$$

where $T_{0}$ is the initial-condition temperature. Since the openloop transfer function, $F(s)$, is $F(s)=F_{1}(s) F_{2}(s)$, any changes in $F_{2}(s)$ change $F(s)$ and modify the closed-loop performance. Changes in $\mathrm{C}$ will occur for various levels and types of liquids, and changes in $\mathrm{R}$ will occur due to pot-lid seating, leakage through the temperature sensor leads, surface on which the pot is placed, etc. 
For a fixed value of input power, i, a time-shared digital computer, shown schematically in Fig. $37 \mathrm{~d}$, can store the temperature data, $\mathrm{T}_{0}, \mathrm{~T}_{1}$, and $\mathrm{T}_{2}$, measured at intervals of time $\Delta \mathrm{t}$ (see Fig. $37 \mathrm{c}$ ). Calculation then yields values for $A=e^{-\Delta t / R C}, R$, and $T_{\infty}=$ final temperature to be reached.

If the final temperature required is $T_{R}$, then the error $T_{R}-T_{\infty}$ can be made zero by changing the magnitude of $i$ to $i+8 i$. That is,

$$
T_{R}-T_{\infty}=R \delta i=\frac{T_{2}-T_{1} A}{i(1-A)},
$$

whence

$$
\delta i=i \frac{\left(T_{R}-T_{\infty}\right)(1-A)}{T_{2}-T_{1} A} .
$$

The change from $i$ to $i+8 i$ is made immediately after measuring $T_{2}$. However, the desired temperature may not be reached for some time due to the time constant, RC. Within the power capabilities of the power source, the temperature $T_{R}$ may be achieved in the time interval $\Delta t$ by magnifying $i+\delta i$ by a factor $k$ for the $\Delta t$ time interval. We have

$$
T_{R}=k(i+8 i) R(1-A)+T_{2} A,
$$

from which,

$$
k=\frac{T_{R}-T_{2} A}{(i+\delta i) R(1-A)} .
$$

The time history of the situation is shown in Fig. 38. After temperature $\mathrm{T}_{\mathrm{R}}$ is reached, the sampling rate would probably be increased so as to rapidly detect and correct for small variations. To summarize:

1. The system has adapted to an arbitrary set of values for $R$ and $C$.

2. It has predicted the final temperature, $T_{\infty}$, for an input $i$ and has used this information and required temperature $T_{R}$ to predict a required in put $\mathrm{i}+\delta_{\mathrm{i}}$.

3. It has calculated a required magnifier, $k$, for $i+\delta i$ and has applied this factor for a time $\Delta t$. The required value of temperature will be achieved in this time.

4. At no time was there a probability of substantial overshoot in temperature. Overshoot is a problem in temperature control because, in many instances, the rate of cooling is significantly less than the available 
rate of heating, and with closed-loop "fast" systems, the response tends to be second-order in nature and temperature overshoot is common. Using openloop programming alleviates this problem.

5. Computer time and complexity of data handling are both minimal. Thus, the computer can be used simultaneously for control of other experiments.

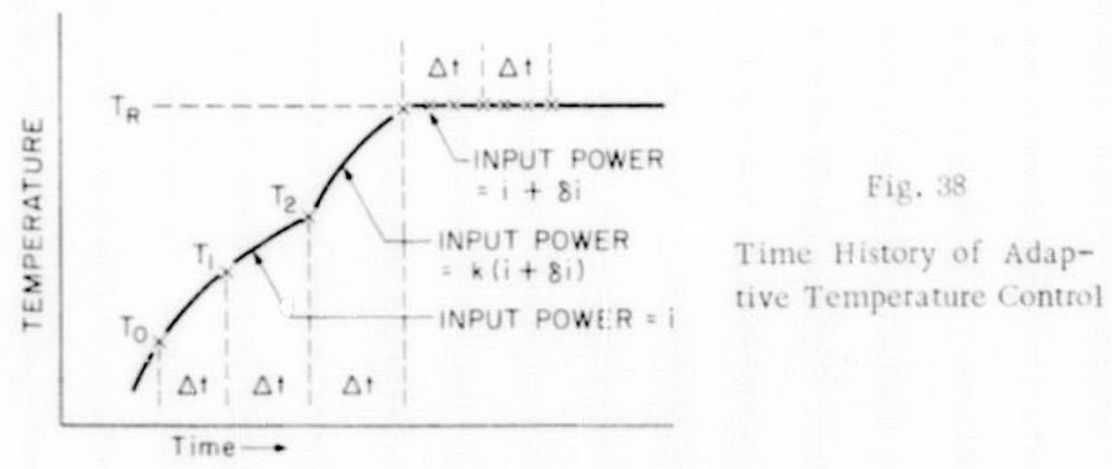

Computer control using prediction techniques can also be applied to second-order systems by using the methods described in Sec. V.B of this report.

\section{B. Marginally Stable Systems}

When $\alpha$ is zero or positive, a system exhibits instability. Also, some systems that are stable for small-signal inputs become unstable for large inputs due to nonlinearities.

For either of these cases, application of the method described in this report can yield stable operation. Automatic measurements of $\psi, \gamma$, and $T_{S}$ and computer calculation of required stabilizing inputs provide for an adaptive situation. The procedure would be most useful during initial testing of aircraft, ships, and other large sophisticated systems where instability might otherwise lead to damage or destruction. Recording of the required error-correction signals could provide the required information for system design improvements.

In Fig. 39, an error $\varepsilon_{0}$ yields an error $\varepsilon_{1}=\epsilon_{0}(1-\psi)$, T $\mathrm{S}$ seconds later. This grows to $\epsilon_{2}=\varepsilon_{1}(1-\psi)$ in another $T_{S}$ seconds and continues to increase unless a correctional input is made. An error-monitor and -control unit can be inserted in the error path as shown and measurements made yielding $\varepsilon_{1}, \varepsilon_{2}$, and $\mathrm{T}_{\mathbf{S}}$. These quantities are applied to the Data Acquisition System (DAS), which feeds back the correctional step, $p$. As shown in the equation for $p$ in Fig. 39, calculation of $\rho$ requires only the values $\varepsilon_{1}$ and $\varepsilon_{2}$ acquired in the time interval $\mathrm{T}_{\mathrm{S}}$. The value for $\mathrm{T}_{\mathrm{S}}$ must be measured for determining the length of time for applying $\rho$. Application of the correctional signal causes the error signal to be reduced smoothly to zero, as shown by the dashed curve. 

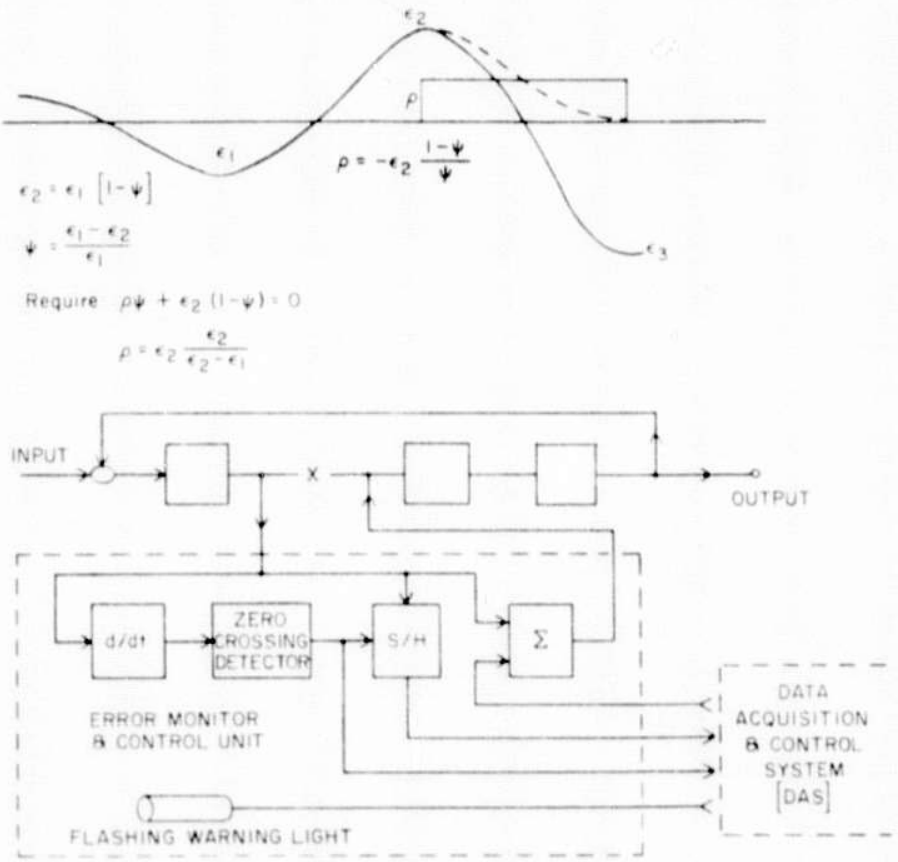

Fig. 39

Possible Error Monitor and Control Unit to Operate with DAS

Because the system is unstable, a new error signal will shortly appear and must be corrected. The DAS can be programmed to detect a requirement for an excess number of error-correcting signals and to cause a warning light to flash, thus signifying instability of the system being monitored.

\section{Large-signal Error Control}

Under certain conditions, an error signal can become so large that the final error-signal amplifiers are saturated. This is illustrated in Fig. 40. An arc in a high-voltage supply causes a large error signal and saturation at the $10^{6} \in$ level. Recovery of control can require a substantial time interval as shown, and the output voltage may swing over wide limits that may cause a new arc. By using signals from earlier stages that are not saturated, the errorcorrection scheme can be implemented and a smooth recovery as shown by the dashed line achieved.

Fig. 40

Large-signal Error Control
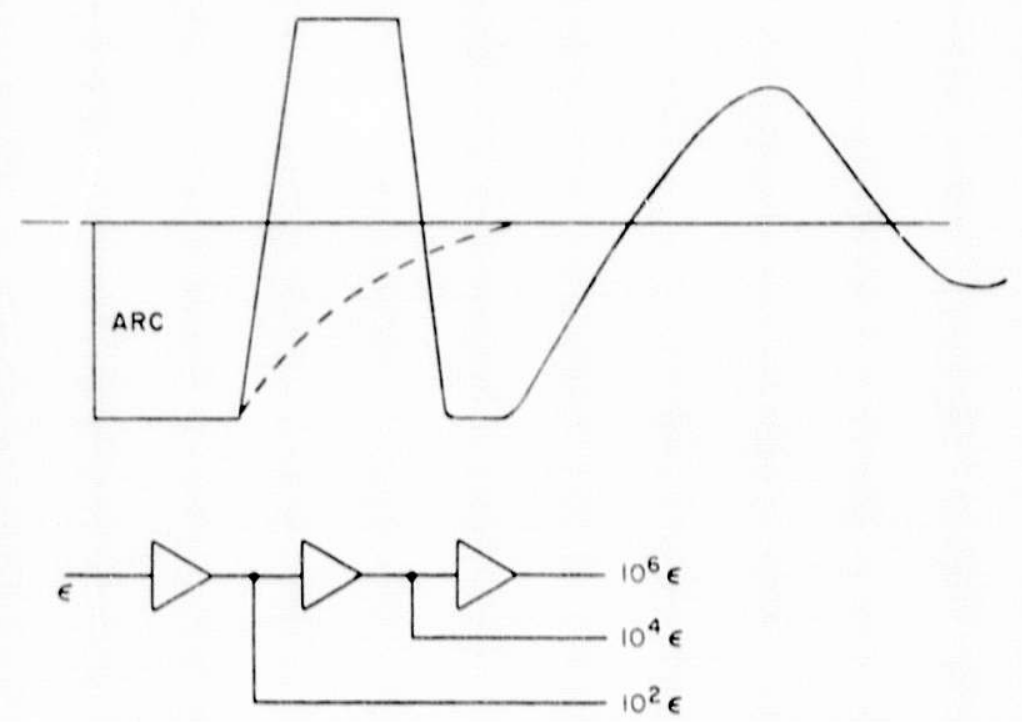


\section{NOISE-GENERATED ERRORS IN SYSTEM RESPONSE}

\section{A. General}

Noise voltages, superimposed on the error signal, limit the ultimate precision of an instrument. Filters must normally be provided to remove some of the noise without adverse effect on the system performance. This is generally the high-frequency portion of the noise spectrum. Included are spike suppressors and noise limiters* for clipping excessively large noise spikes. The low-frequency components of the noise then give the most trouble.

The most common criterion for improved performance is to attempt to minimize the time average of the root-mean-square error of the system output. We will consider here the case in which system output error is due to the internal noise of the system. That is, we will assume that the input command is both noise-free and of constant value.

\section{B. Mean-square Error}

For simplicity, we will deal here with the mean-square error, written as $\epsilon^{2}$. The root-mean-square error is the square root of this quantity and is generally known as the standard deviation, $\sigma$. We will assume the case of stationary time series; i.e., the average noise spectrum remains approximately constant from minute to minute and from day to day. The power spectral density is a plot of the expected average noise power at each frequency. One such plot, shown in Fig. $4 \mathrm{la}$, indicates that, for this case, the noise power is greater at some frequencies than at others.

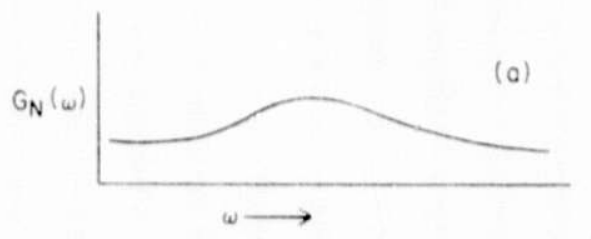

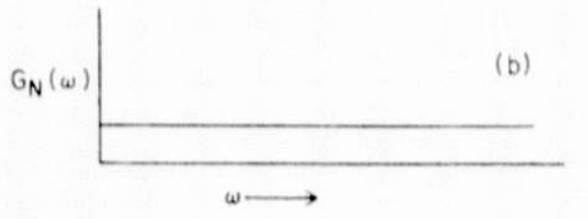

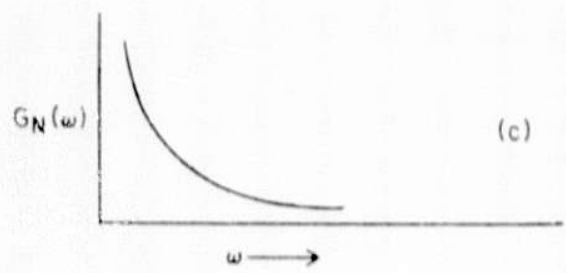

Fig. 41

Typical Examples of Noise Spectral Densities. (a) General noise spectrum; (b) "White" noise spectrum; and (c) $1 / \mathrm{f}$ noise spectrum.

* See, for example, Appendix B of Ref, 5 . 
Shown in Fig. 4lb, is a plot of a "flat" noise spectrum. This is termed "white" noise. Thermal noise in a resistor is one example of white noise. Shown in Fig. $41 \mathrm{c}$ is an example of "l/f" noise. This noise characteristic is observed for vacuum tubes and certain solid-state devices.

The mean-square noise power (as would be read on a long-term averaging wattmeter) can be related to the power-spectrum density by

$$
\overline{\epsilon_{\mathrm{n}}^{2}}=\int_{0}^{\infty} \mathrm{G}_{\mathrm{n}}(\omega) \mathrm{d} \omega
$$

When the noise is applied to the input of a system with frequency response $F(j \omega)$, the output mean-square error will be

$$
\overline{\epsilon_{0}^{2}}=\int_{0}^{\infty}[F(j \omega)]^{2} G_{n}(\omega) d \omega .
$$

It is of interest to calculate the mean-square error for the elementary secondorder system for the case of white noise. From Fig. 21, the frequency response of the second-order system is

$$
F(j \omega)=\frac{1}{\sqrt{\left(1-\omega^{2} / \omega_{n}^{2}\right)^{2}+\omega^{2} \tau^{2}}} .
$$

Therefore, since $G_{n}(\omega)=G_{0}$ for any $\omega$, we have (see, for example, Ref. 6, p. 129)

$$
\overline{\epsilon_{0}^{2}}=G_{0} \int_{0}^{\infty} \frac{d \omega}{\left(1-\omega^{2} / \omega_{n}^{2}\right)^{2}+\omega_{n}^{2} \tau^{2}}=\pi G_{0} \frac{\omega_{n}}{4 \zeta} .
$$

This result can also be obtained ${ }^{5}$ by using autocorrelation functions. The autocorrelation of white noise with a constant spectral density $\mathrm{G}_{0}$ is

$$
\varphi_{n}(\tau)=\pi G_{0} \delta(\tau)
$$

For a system with weighting function $F(\tau)$, the output mean-square error due to white-noise input will be

$$
\overline{\varepsilon_{0}^{2}}=\varphi(0)=\pi \mathrm{G}_{0} \int_{0}^{\infty}[F(\tau)]^{2} \mathrm{~d} \tau .
$$

The second-order system of Fig. 3 has the weighting function

$$
F(t)=\frac{\omega_{n}^{2}}{\omega} e^{-\alpha t} \sin \omega t .
$$


Therefore,

$$
\begin{aligned}
\overline{\epsilon_{0}^{2}} & =\varphi(0)=\pi G_{0} \int_{0}^{\infty} \frac{\omega_{n}^{4}}{\omega^{2}} e^{-2 \alpha \tau} \sin ^{2} \omega \tau d \tau \\
& =\pi G_{0} \frac{\omega_{n}}{4 \zeta},
\end{aligned}
$$

which agrees with the results obtained by Eq. 50 .

According to Sec. V.A.2, changing the loop gain of a second-order system has the effect of changing the capacitance of the circuit of Fig. 3. The result of gain change is shown in Table I. Some of the data is repeated in Table IV, along with the factor $\omega_{n} /(45)$ and the expected standard deviation of the mean, which is

$$
\sigma=\sqrt{\overline{\epsilon^{2}}}=\sqrt{\varphi(0)}
$$

TABLE IV. Noise Response of the $F_{0}(s)$ System

\begin{tabular}{ccccccc}
\hline A & $\omega_{\mathrm{n}}$ & $\omega$ & $\zeta$ & $\psi$ & $\overline{\epsilon_{0}^{2}} \sim \omega_{\mathrm{n}} /(4 \zeta)$ & $\sigma \simeq \sqrt{\epsilon_{0}^{2}}$ \\
\hline 50 & 7.07 & 5.0 & 0.707 & 1.043 & 2.5 & 1.58 \\
100 & 10.0 & 8.66 & 0.5 & 1.17 & 5.0 & 2.24 \\
400 & 20.0 & 19.36 & 0.25 & 1.43 & 20.0 & 4.47 \\
\hline
\end{tabular}

Table IV shows that increasing loop gain from 50 to 400 increases the bandwidth by a factor of 4 and the step overshoot from 4.3 to $43 \%$. The mean-square error is increased by $20 / 2.5=8$ and the standard deviation by $4.47 / 1.58=2.83$. Thus, while the system step response has been improved by an order of magnitude due to gain increase (plus application of the theory) as shown in Sec. V.A.3, the output standard deviation due to white noise will be increased by a factor of 2.83 .

C. Method for the Reduction of System Output Error due to Internal Noise

\section{Application}

A family of fast-response fuel-rod calorimeters has been developed at ANL for the nondestructive measurement of alpha activity of nuclear fuel. ${ }^{7-10}$ The equation mechanized in the instruments is

$$
\mathrm{W}=\mathrm{K}\left(\mathrm{T}_{3}-\mathrm{T}_{2}\right) \text { watts }
$$

where $T_{3}$ is the temperature of the inner (sample) cylinder, $T_{2}$ is the temperature of a surrounding (reference) cylinder, $\mathrm{K}$ is the thermal conductivity between the cylinders, and $W$ is the power required to maintain the temperature 
difference $T_{3}-T_{2}$. With a depleted (nonradioactive) sample inserted, the electrical power $W_{0}$ is measured. The power is the result of an amplified error signal and contains noise because of the very high gain required to control $\mathrm{T}_{3}$ to a precise temperature, $\sigma\left(\mathrm{T}_{3}\right) \leq 50 \mu \mathrm{K}(0.000050 \mathrm{~K})$. Therefore, $\bar{W}_{0}$ is the mean of a number, $n$, of readings and can be expressed with an accuracy of $\overline{W_{0}} \pm \sigma\left(\overline{W_{0}}\right)$, where $\sigma\left(\overline{W_{0}}\right)$ is the standard deviation (rms error) of the mean.

When a rod containing alpha activity is inserted, the heat produced by the alpha particles impacting on the inside of the rod casing provides part of the power for heating. Hence, the required electrical power is reduced and is measured as $\overline{W_{e}} \pm \sigma\left(\overline{W_{e}}\right)$. The equivalent heating power, $W_{\alpha}$, due to the alpha activity is then determined as

$$
\mathrm{W}_{\alpha}=\overline{\mathrm{W}_{0}}-\overline{\mathrm{W}_{\mathrm{e}}} \pm \sqrt{\left[\sigma\left(\overline{\mathrm{W}_{0}}\right)\right]^{2}+\left[\sigma\left(\overline{\mathrm{W}_{\mathrm{e}}}\right)\right]^{2}}
$$

The number of grams of fuel can then be determined by a program that requires information on the isotopic content of the fuel and the specific power for each isotope. ${ }^{7}$

Equation 55 indicates that small values for $\sigma\left(\overline{\mathrm{W}_{0}}\right)$ and $\sigma\left(\overline{\mathrm{W}_{\mathrm{e}}}\right)$ are desirable. It is also evident from Eq. 48 and from probability theory that there is a lower limit on the value of $\sigma=\sqrt{\sqrt{\varepsilon^{2}}}$ to be obtained. That is, measurement for 2 min will probably yield a smaller $\sigma$ than will measurement for 1 min; however, measurement for $2 \mathrm{~h}$ may yield a value for $\sigma$ no smaller than that obtained by measurement for $1 \mathrm{~h}$.

It was decided to apply the control theory developed in this report to the fNL Model IV calorimeter. ${ }^{9,10}$ The improvements in performance sought were:

a. A faster scttling time after insertion of a sample.

b. Automatic indication of the fact that the system output error is sufficientiy small that a measurement may be made.

c. Control of the error signal due to noise in such a manner as to reduce the standard deviation of the output error.

\section{Method}

The closed-loop error-correction method of Sec. V.B was used. As shown in Fig. 42, it was so arranged that the error-correction unit could

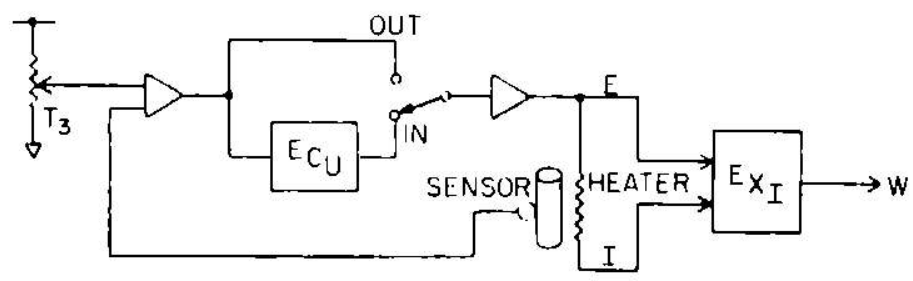

Fig. 42

Inclusion of Error-correction Init (LC:L) in Feesback Patl 
be switched in or out of the system, thus allowing for comparison of system performance with and without inclusion of the unit.

Figure 43 is a block diagram of the error-correction unit. The error signal is applied to the input summing circuit and also to the in/out switch contact. A second input to the input summer is a bias variable over $\pm 15 \mathrm{~V}$. The same bias is applied to the output summer. The net effect is that the bias level between the summers can be changed but there is no net bias change between $E_{i n}$ and $E_{\text {out }}^{\prime}$.

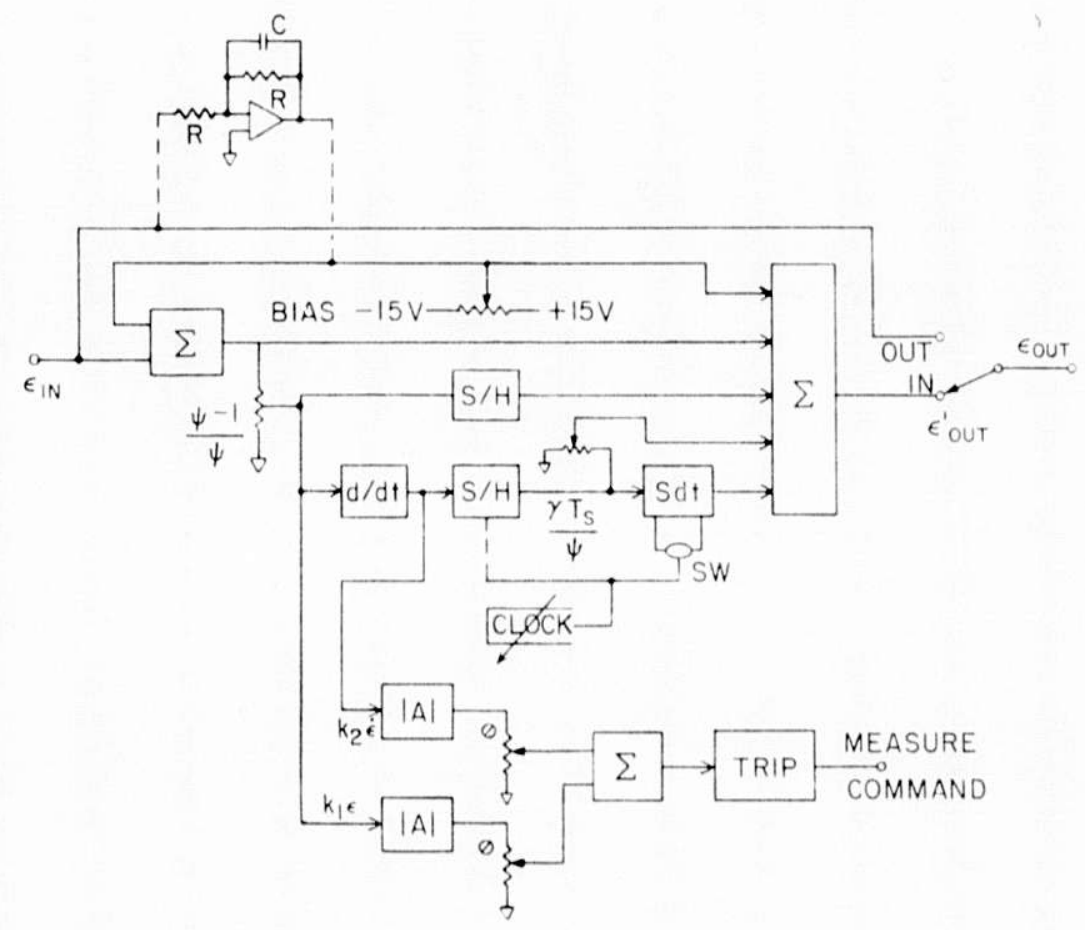

Fig. 43. Block Diagram of Error-correction Unit

An alternative method, which is also automatic, is to use an inverting amplifier with a long time constant RC shown connected by dashed lines. This keeps the average bias between summers at a value of zero. In this case, the bias pot is not used. The output of the input summer is applied directly to the output summer and is also modified in value by a factor $(\psi-1) / \psi$. A clock pulse operates sample/hold units to acquire $\epsilon(\psi-1) / \psi$ and $\dot{\epsilon}(\psi-1) / \psi$, the former being applied to the output summer and the latter being modified by $\gamma \mathrm{T}_{\mathrm{S}} / \psi$ before application to the output summer.

The signal $\dot{\epsilon}(\psi-1) / \psi$ is also applied to a ramper whose output is fed to the output summer and is reset to zero at each clock pulse. [It was discovered that the clock pulse interval should be much shorter (by at least a factor of 5) than the interval $T_{S}$ used in Sec. V.]

The signals $\mathrm{k}_{1} \dot{\epsilon}$ and $\mathrm{k}_{2} \epsilon$ are applied to absolute-amplitude amplifiers whose outputs will always be positive, regardless of the input polarity. Their 
outputs are adjusted in magnitude, summed, and applied to a trip unit. From Fig. 16, it is seen that the zero values for $\dot{\varepsilon}$ coincide approximately with maximum values for $\epsilon$ and vice versa. Thus requiring that both $\epsilon$ and $\dot{\epsilon}$ have small values before beginning a measurement proves to be a good criterion.

A schematic of the error-correction unit is shown in Fig. 44.

\section{Results}

Figure 45 shows a pen recording of the amplified error signal. The peaks span about $\pm 0.00004^{\circ} \mathrm{C}$. The first 5 min of the trace was made with the error-correction unit out of the circuit. For 150 samples, the mean was 974.50 and the standard deviation of the mean was 0.455 . 'This provides a percentage standard deviation of the mean at one sigma of $0.047 \%$.

The second 5 min was made with the error-corection unit in the circuit. Here the mean is 974.527, and the standard deviation of the mean is 0.0818 . This provides a percentage standard deviation of the mean at one sigma of about $0.0084 \%$, an improvement over the above by a factor of 5.6 for the same number of samples.

Only item "c" of Sec. X.C. I has been described in this section. Items "a" and "b" were also favorable.

\section{Analysis of Results}

A theorem due to Campbel1 ${ }^{11,12}$ states that if the response, $F(t)$, of a system to an input pulse $\varepsilon$ is

$$
A(t)=\delta F(t)
$$

then its mean response to a random series of pulses occurring at a mean rate $f$ is

$$
\bar{\theta}=\int \delta \int_{0}^{\infty} F(t) d t
$$

and the mean-squared error output will be

$$
\overline{\Delta A^{2}}=\mathrm{f} \delta^{2} \int_{0}^{\infty}[\mathrm{F}(\mathrm{t})]^{2} \mathrm{dt}
$$

(Equation 56 may be compared with Eq. 51.)

This result implies that error correction for noise pulses will be more effective if a higher clock rate for the system of Fig. 44 is used. 


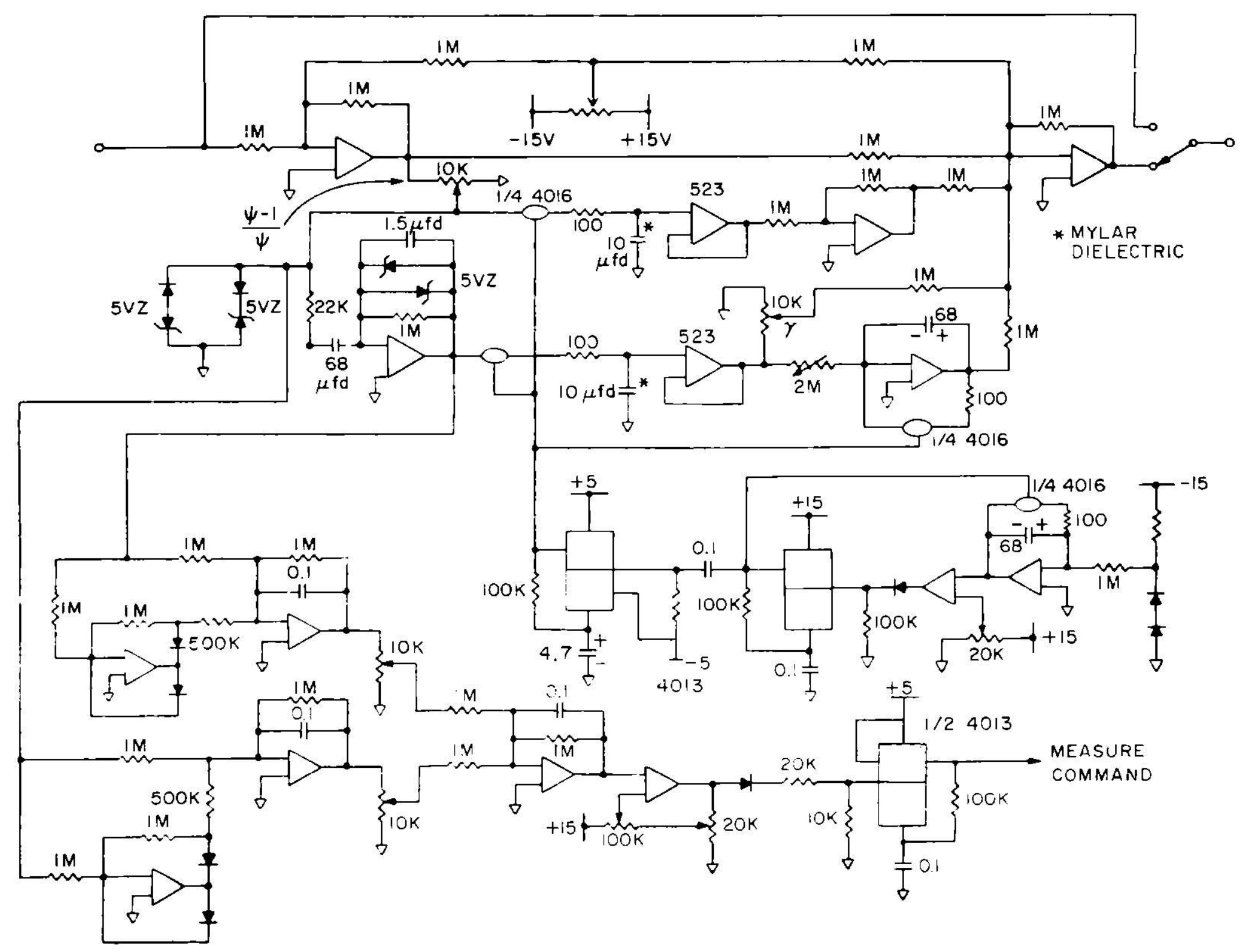

Fig. 4t. Schematic Diagram of Error-correction Lnit 


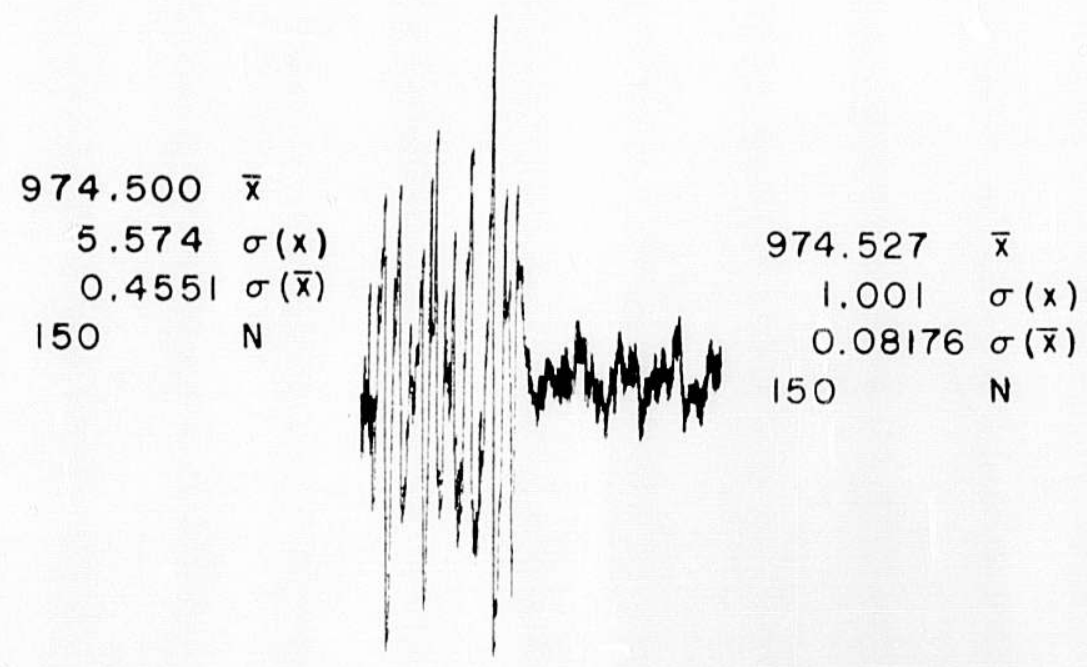

Fig. 45. Recording of Amplified Error Signal of Precision Calorimeter. First half is normal operation; peak-to-peak error is about $+0.00004^{\circ} \mathrm{C}$. Second half is operation with error-correction unit in operation.

That is, to compare $\overline{\epsilon_{0}^{2}}$ for a system with and without the error-correction unit (ECU),

$$
\frac{\overline{\varepsilon_{0}^{2}}(\mathrm{ECU})}{\overline{\epsilon_{0}^{2}}} \approx \frac{1}{\mathrm{f}},
$$

where $f$ is the clock rate.

We can also write

$$
\frac{\overline{\epsilon^{2}}(E C U)}{\overline{\epsilon^{2}}} \simeq \frac{\int_{0}^{\pi / \omega}\left[F^{\prime}(t)\right]^{2} d t}{\int_{0}^{\infty}[F(t)]^{2} d t}
$$

where $F^{\prime}(t)$ is the effective system weighting function due to use of the errorcorrection unit, since the output due to an input pulse, $\delta$, is the sum of the normal response to the pulse minus the response to a step, plus the response to a ramp. Figure 46 compares $F(t)$ with $F^{\prime}(t)$. Numerical integration yields

$$
\frac{\int_{0}^{\pi / \omega}\left[F^{\prime}(t)\right]^{2} d t}{\int_{0}^{\infty}[F(t)]^{2} d t} \sim \frac{1}{3} \text {. }
$$

Using Eqs. 59 and 57, we obtain

$$
\frac{\overline{\epsilon_{0}^{2}}(\mathrm{ECU})}{\overline{\epsilon_{0}^{2}}} \simeq \frac{1}{3 \mathrm{f}}
$$


where $f$ is the number of samples per half cycle, $T_{S}$. In the above case, $f \simeq 10 ;$ thus the expected improvement would be

$$
\frac{\overline{\epsilon_{0}^{2}}(E C U)}{\overline{\epsilon_{0}^{2}}} \simeq \frac{1}{3 \times 10} \simeq \frac{1}{30} .
$$

The standard deviation is the square root of the mean-square error. Thus,

$$
\frac{\sigma(E C U)}{\sigma} \simeq \frac{1}{\sqrt{30}} \simeq \frac{1}{5.5},
$$

which approximately agrees with the improvement observed.

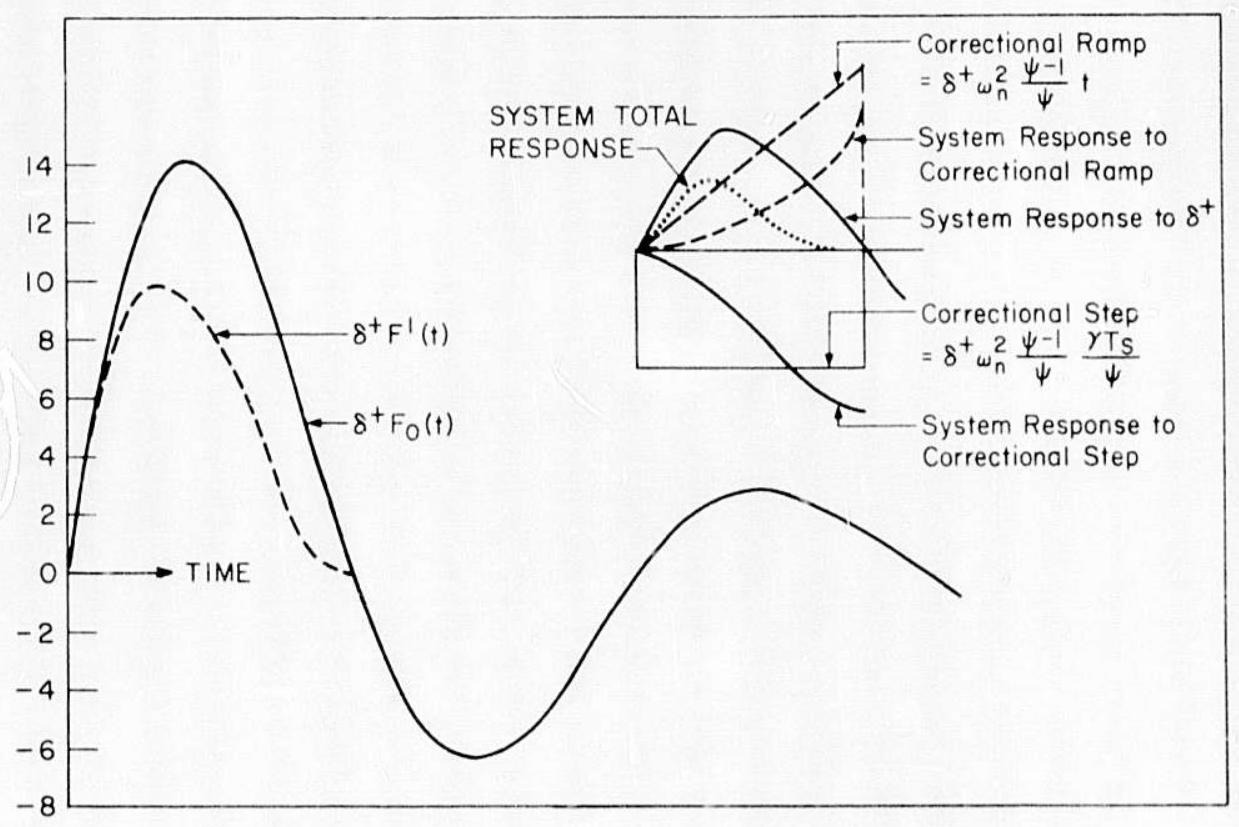

Fig. 46. Impulse Response of $\mathrm{F}_{\mathrm{C}}(\mathrm{s})$ System. $\delta \mathrm{F}_{0}(\mathrm{t})$ is the normal response, and $\delta F^{\prime}(t)$ is the response when using the error-correction unit.

\section{Discussion of Results}

Without the error-correction unit, the standard deviation of the mean would not have been reduced significantly for longer sample times. This follows from Eq. 48 and from experience. With the error-correction unit in operation, the standard deviation of the mean was immediately improved by a factor of more than 5 for the same number of samples. Perhaps a more important point is that a quantity could have been measured to a prescribed precision in a much shorter time interval through use of this method. 
Many systems are designed to yield an output that is a scaler value of an internal reference. For example, a high-voltage power supply has an output

$$
E_{O}=M E_{R e f},
$$

where $M$ may be variable. For a high-gain system with a clean reference source, output noise is due to thermal noise, output loading, and line transients. The device described above will substantially reduce such output noise below the value normally obtainable. 
EPILOGUE

"Would you tell me please," said Alice, "which way I ought to go from here?"

"That depends a great deal on where you want to get to," said the Cat.

"I don't much care where ...," said Alice.

"Then it doesn't matter which way you go," said the Cat.

"...So long as I get somewhere," Alice added as an explanation.

"Oh, you're sure to do that," said the Cat, "if you only walk long enough." 


\section{APPENDIX}

\section{Effect of Pole and Zero Locations on Time Response of Systems}

The effect of pole and zero locations on systems time response can be more easily visualized by use of s-plane geometry. Figure A.la shows a system with a pair of complex conjugate poles, located at $-\alpha \pm j \omega$. The natural frequency, $\omega_{n}$, is the radial distance from the origin to either pole with an angle $\varphi$ between the $\omega_{n}$ vector and the negative real axis. It is seen that the step response can be described in terms of $\omega_{n}, \omega, \alpha$, and $\varphi$, and with some practice, the effect of moving the poles about in the s-plane can be visualized.

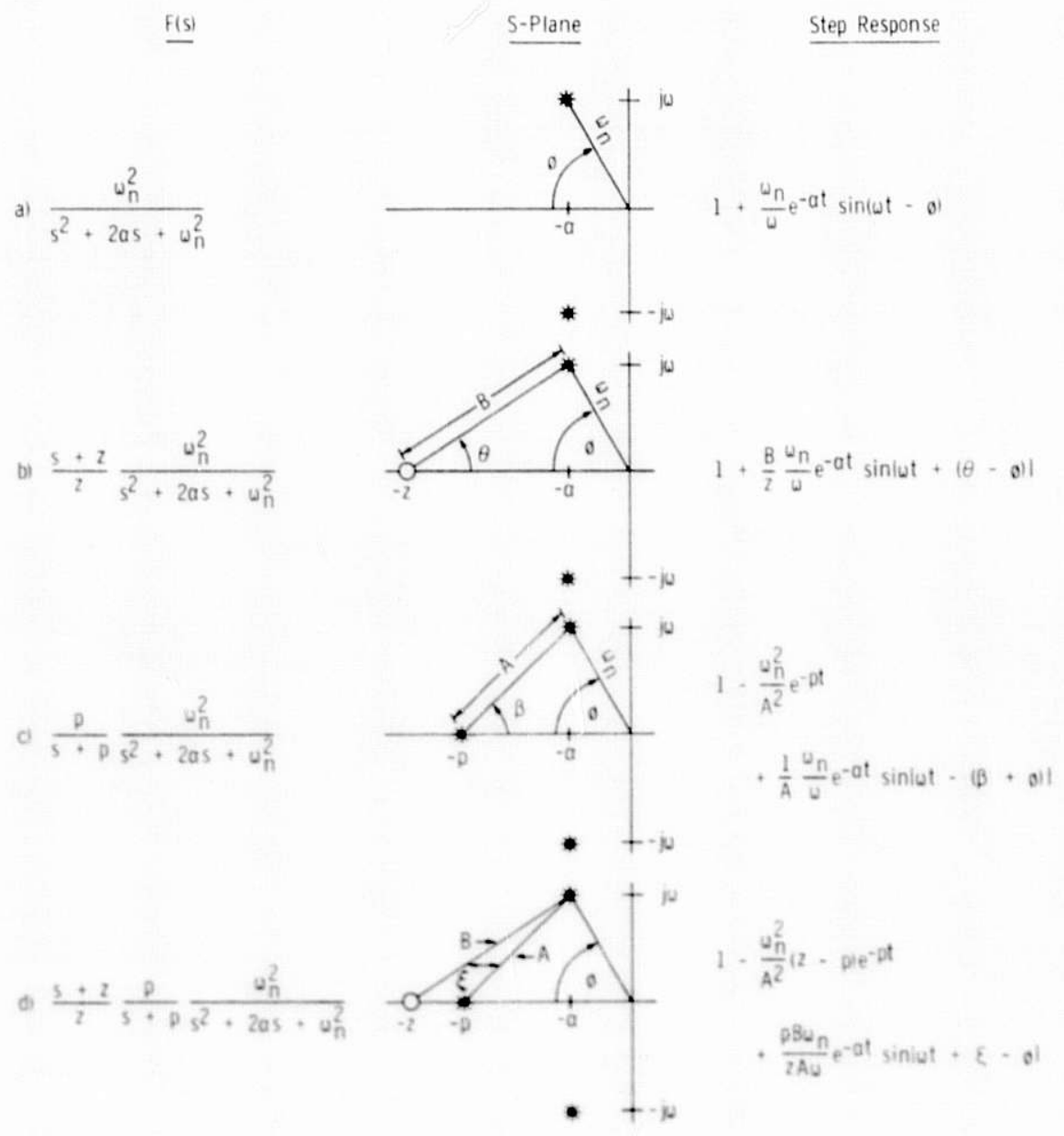

Fig. A.1. s-plane Geometry and Step Response for Several Systems

In Fig. A. Ib, a real zero, $z$, has been added. The distance between the zero and either complex pole is $B$, with the vector $B$ making an angle $\theta$ with respect to the negative real axis. As the zero is moved closer to the origin, the transient term becomes large, due to the multiplier $\mathrm{B} / \mathrm{z}$. The zero has modified the amplitude and phase angle, but has not changed the form of the time function. 
In Fig. A. lc, a real pole, p, has been added to the system of Fig. A.la. The distance between the real and complex poles is the vector $A$, which forms an angle $\beta$ with respect to the negative real axis. The effect of the real pole is to modify the oscillatory term by the factor $1 /$ A (generally a reduction in amplitude) and to give rise to a first-order exponential term.

In Fig. A.ld, both a real pole and a real zero have been added to the system of Fig. A.la. It is seen that the first-order exponential term is multiplied by $z$ - p. This shows that for a dipole $(z=p)$, the exponential term disappears, $A=B$, and $\xi=0$. Thus the response is the same as for the system of Fig. A.la.

For higher-order systems, computer modeling is valuable for "optimizing" the transfer function. For example, Ref. 13 is an investigation of eight mathematical criteria for "optimizing" systems through the eighth order. The methods described in the present report yield a more error-free response than do any of those investigated in Ref. 13. 


\section{ACKNOW LEDGMENTS}

I am grateful to Stanley J. Rudnick, Robert J. Epstein, and John Gilroy, present and past Directors of the Argonne National Laboratory Electronics Division, for their enthusiastic support of the work described here.

Special gratitude is due Donald P. Reynolds, Chicago Operations Office, U. S. Department of Energy, for many helpful discussions on control-systems theory.

\section{REFERENCES}

1. Benjamin C. Kuo, Automatic Control Systems, Prentice-Hall, Inc., Englewood Cliffs, N.J. (1962).

2. J. G. Truxa1, Editor, Control Engineers' Handbook, McGraw-Hill Book Company, New York (1958).

3. C. J. Savant, Jr., Basic Feedback Control System Design, McGraw-Hill Book Company, New York (1958).

4. J. G. Truxa1, Automatic Feedback Control System Synthesis, McGraw-Hill Book Company, New York (1955).

5. J. H. Laning, Jr., and R. H. Battin, Random Processes in Automatic Control, McGraw-Hill Book Company, New York (1956).

6. H. S. Tsein, Engineering Cybermetics, McGraw-Hill Book Company, New York (1954).

7. N. S. Beyer, R. B. Perry, R. W. Brandenburg, and R. N. Lewis, Four Passive Assay Techniques Applied to Mixed-oxide Fuel, ANL-7906 (Jan 1972).

8. N. S. Beyer, R. N. Lewis, and R. B. Perry, Fast Response Fuel Rod Calorimeter with a 36 -inch Fuel Column Capacity, Nuc1. Mater. Manage. 3, 118-130 (1974).

9. S. B. Brumbach, A. M. Finkbeiner, R. N. Lewis, and R. B. Perry, Plutonizm Calorimetry and SNM Holdup Measurements: Progress Report for the Period March 1976-August 1976, ANL-77-8, NUREG-0182 (Feb 1977).

10. N. S. Beyer, R. N. Lewis, and R. B. Perry, Dry Portable Calorimeter for Non-destmuctive Measurement of the Activity of Nuclear Fuel, U. S. Patent 3,995,485 (Dec 7, 1976).

11. F. N. H. Robinson, Noise in Electrical Circuits, Oxford University Press (1962).

12. N. R. Campbe11, Proc. Camb. Phil. Soc. 15, 117-136, 310-328 (1909).

13. D. Graham and R. C. Lathrop, The Synthesis of "Optimum" Transient Response: Criteria and Standard Forms, Trans. Am. Instit. Electr. Eng. Part 272 , 273-288 (1953). 
Distribution of ANL-78-14

Internal:
J. A. Kyger
C. T. Roche
E. A. Jung
R. Avery
S. B. Erumbach
W. C. Kaiser
L. Burris
W. F. Henning
G. J. Kamis
D. W. Cissel
G. T. Garvey
R. M. Kash
S. A. Davis
G. D. Ansley
D. J. Keefe
B. R. T. Frost
E. E. Armand
F. R. Lenkszus
D. C. Rardin
J. A. Bjorkland
A. A. Longnecker
R. J. Teunis
J. L. Blahunka
C. E. Till
J. P. Bobis
R. S. Zeno
D. W. Bradley
R. Brenner
H. O. Monson
J. Brewer
R. N. Lewis (50)
W. K. Brookshier
F. J. Lynch
W. W. Managan
W. P. Mc Dowell
P. R. Fields
M. J. Cattelino
M. K. McGee
N. S. Beyer
R. T. Daly
R. Brandenburg
R. J. DeLand
O. D. Despe
P. E. Neal
D. N, Eggenberger
R. J. Epstein
S. J. Rudnick (5)
J. J. Eichholz
R. B. Perry
V. J. Elsbergas
L. M. Bollinger
W. R. Erdman
G. A. McGinnis
J. E. Miranda
C. A. Nelson
D. R. Petterson
J. M. Paul
R. J. Pecina
L. L. Prucha
M. W. Schultz
I. S. Sherman
R. L. Smith
A. Langsciorf
R. W. Fergus
M. G. Strauss
F. R. George
D. J. Travis
S. L. Halverson
J. A. Wiesemes
G. Popper
J. R. Haumann
L. Amiot
G. T. Hicks
R. A. Noland
R. W. Howard
D. P. Reynolds
E. W. Johanson
C. M. Stevens
D. C. Knoop
L. Van Loon
C. B. Morgan
A. L. Winiecki
A. B. Krisciunas ANL Contract File ANL Libraries (5) TIS Files (6)

External:

DOE-TIC, for distribution per UC-37 and -38 (224)

Manager, Chicago Operations Office

Chief, Office of Patent Counsel, $\mathrm{CH}$

President, Argonne Universities Association 